Revised version

February 2014

\title{
EFFECTS OF AN EXTRA $U(1)$ AXIAL CONDENSATE ON THE STRONG DECAYS OF PSEUDOSCALAR MESONS
}

\author{
Enrico Meggiolard® \\ Dipartimento di Fisica, Università di Pisa, and INFN, Sezione di Pisa, \\ Largo Pontecorvo 3, I-56127 Pisa, Italy.
}

\begin{abstract}
We consider a scenario (supported by some lattice results) in which a $U(1)$-breaking condensate survives across the chiral transition in QCD. This scenario has important consequences for the pseudoscalar-meson sector, which can be studied using an effective Lagrangian model. In particular, generalizing the results obtained in two previous papers, where the effects on the radiative decays $\eta, \eta^{\prime} \rightarrow \gamma \gamma$ were studied, in this paper we study the effects of the $U(1)$ chiral condensate on the strong decays of the "light" pseudoscalar mesons, i.e., $\eta, \eta^{\prime} \rightarrow 3 \pi^{0} ; \eta, \eta^{\prime} \rightarrow \pi^{+} \pi^{-} \pi^{0} ; \eta^{\prime} \rightarrow \eta \pi^{0} \pi^{0} ; \eta^{\prime} \rightarrow \eta \pi^{+} \pi^{-}$; and also on the strong decays of an exotic ("heavy") $S U(3)$-singlet pseudoscalar state $\eta_{X}$, predicted by the model.

PACS numbers: 12.38.Aw, 11.15.Pg, 11.30.Rd, 12.39.Fe
\end{abstract}

*E-mail: enrico.meggiolaro@df.unipi.it 


\section{Introduction}

It is well known that the QCD vacuum has a very complicated structure, characterized by some non-trivial local (or also non-local) condensates, whose behaviour as a function of the temperature $T$ also characterizes the phase structure of the theory.

For example, a phase transition which occurs in QCD at a finite temperature $T_{c h}$ is the restoration of the $S U(L) \otimes S U(L)$ chiral symmetry (in association with $L=2,3$ massless quarks), which for $T<T_{c h}$ is broken spontaneously by the non-zero value of the so-called "chiral condensate", i.e., $\langle\bar{q} q\rangle \equiv \sum_{i=1}^{L}\left\langle\bar{q}_{i} q_{i}\right\rangle[1]$. But QCD with $L$ massless quarks has also (at least at the classical level) a $U(1)$ axial symmetry [2, 3]. This symmetry is broken by an anomaly at the quantum level, which in the "Witten-Veneziano mechanism" [4, 5] plays a fundamental role (via the so-called "topological susceptibility") in explaining the large mass of the $\eta^{\prime}$ meson. The role of the $U(1)$ axial symmetry for the finite temperature phase structure has been so far not well clarified. One expects that, above a certain critical temperature $T_{U(1)}$, also the $U(1)$ axial symmetry will be (effectively) restored but it is still unclear whether $T_{U(1)}$ has or has not something to do with $T_{c h}$.

In this paper we re-consider a scenario (which was originally proposed in Refs. 6, [7, 8, 9] and elaborated in Refs. [10, 11, 12], and which seems to be supported by some lattice results on the so-called "chiral susceptibilities" [13, 14, 15]) in which a new $U(1)-$ breaking condensate survives across the chiral transition at $T_{c h}$, staying different from zero up to a temperature $T_{U(1)}>T_{c h} . T_{U(1)}$ is, therefore, the temperature at which the $U(1)$ axial symmetry is (effectively) restored, meaning that, for $T>T_{U(1)}$, there are no $U(1)-$ breaking condensates. The new $U(1)$ chiral condensate has the form $C_{U(1)}=\left\langle\mathcal{O}_{U(1)}\right\rangle$, where, for a theory with $L$ light quark flavours, $\mathcal{O}_{U(1)}$ is a $2 L$-fermion local operator that has the chiral transformation properties of [3, 16, 17]:*

$$
\mathcal{O}_{U(1)} \sim \operatorname{det}_{s t}\left(\bar{q}_{s R} q_{t L}\right)+\operatorname{det}_{s t}\left(\bar{q}_{s L} q_{t R}\right)
$$

where $s, t=1, \ldots, L$ are flavour indices. The colour indices [not explicitly indicated in Eq. (1.1)] are arranged in such a way that: i) $\mathcal{O}_{U(1)}$ is a colour singlet, and ii) $C_{U(1)}=\left\langle\mathcal{O}_{U(1)}\right\rangle$ is a genuine $2 L$-fermion condensate, i.e., it has no disconnected part proportional to some power of the quark-antiquark chiral condensate $\langle\bar{q} q\rangle$ : the explicit form of the condensate

*Throughout this paper we use the following notations for the left-handed and right-handed quark fields: $q_{L, R} \equiv \frac{1}{2}\left(1 \pm \gamma_{5}\right) q$, with $\gamma_{5} \equiv-i \gamma^{0} \gamma^{1} \gamma^{2} \gamma^{3}$. 
for the cases $L=2$ and $L=3$ is discussed in detail in Appendix A (see also Refs. [8, 9, 10]).

This scenario has important consequences for the pseudoscalar-meson sector. The low-energy dynamics of the pseudoscalar mesons, including the effects due to the anomaly, the $q \bar{q}$ chiral condensate and the new $U(1)$ chiral condensate, can be described, in the limit of large number $N$ of colours, and expanding to the first order in the light quark masses, by an effective Lagrangian written in terms of the topological charge density $Q$, the mesonic field $U_{i j} \sim \bar{q}_{j R} q_{i L}$ (up to a multiplicative constant) and the new field variable $X \sim \operatorname{det}\left(\bar{q}_{s R} q_{t L}\right)$ (up to a multiplicative constant), associated with the new $U(1)$ condensate [6, 7, 8, 10]:

$$
\begin{gathered}
\mathcal{L}\left(U, U^{\dagger}, X, X^{\dagger}, Q\right)=\frac{1}{2} \operatorname{Tr}\left(\partial_{\mu} U \partial^{\mu} U^{\dagger}\right)+\frac{1}{2} \partial_{\mu} X \partial^{\mu} X^{\dagger} \\
-V\left(U, U^{\dagger}, X, X^{\dagger}\right)+\frac{i}{2} \omega_{1} Q \operatorname{Tr}\left(\ln U-\ln U^{\dagger}\right) \\
+\frac{i}{2}\left(1-\omega_{1}\right) Q\left(\ln X-\ln X^{\dagger}\right)+\frac{1}{2 A} Q^{2},
\end{gathered}
$$

where the potential term $V\left(U, U^{\dagger}, X, X^{\dagger}\right)$ has the form:

$$
\begin{array}{r}
V\left(U, U^{\dagger}, X, X^{\dagger}\right)=\frac{\lambda_{\pi}^{2}}{4} \operatorname{Tr}\left[\left(U^{\dagger} U-\rho_{\pi} \mathbf{I}\right)^{2}\right]+\frac{\lambda_{X}^{2}}{4}\left(X^{\dagger} X-\rho_{X}\right)^{2} \\
-\frac{B_{m}}{2 \sqrt{2}} \operatorname{Tr}\left(M U+M^{\dagger} U^{\dagger}\right)-\frac{c_{1}}{2 \sqrt{2}}\left[\operatorname{det}(U) X^{\dagger}+\operatorname{det}\left(U^{\dagger}\right) X\right] .
\end{array}
$$

$M=\operatorname{diag}\left(m_{1}, \ldots, m_{L}\right)$ is the quark mass matrix and $A$ is the topological susceptibility in the pure Yang-Mills (YM) theory. (This Lagrangian generalizes the one originally proposed in Refs. [18, 19, 20, 21, 22], which included only the effects due to the anomaly and the $q \bar{q}$ chiral condensate.) All the parameters appearing in the Lagrangian must be considered as functions of the physical temperature $T$. In particular, the parameters $\rho_{\pi}$ and $\rho_{X}$ determine the expectation values $\langle U\rangle$ and $\langle X\rangle$ and so they are responsible respectively for the behaviour of the theory across the $S U(L) \otimes S U(L)$ and the $U(1)$ chiral phase transitions, as follows:

$$
\begin{aligned}
\left.\rho_{\pi}\right|_{T<T_{c h}} & \equiv \frac{1}{2} F_{\pi}^{2}>0,\left.\quad \rho_{\pi}\right|_{T>T_{c h}}<0 \\
\left.\rho_{X}\right|_{T<T_{U(1)}} & \equiv \frac{1}{2} F_{X}^{2}>0,\left.\quad \rho_{X}\right|_{T>T_{U(1)}}<0 .
\end{aligned}
$$

The parameter $F_{\pi}$ is the well-known pion decay constant, while the parameter $F_{X}$ is related to the new $U(1)$ axial condensate. Indeed, from Eq. (1.4), $\rho_{X}=\frac{1}{2} F_{X}^{2}>0$ 
for $T<T_{U(1)}$, and therefore, from Eq. (1.3), $\langle X\rangle=F_{X} / \sqrt{2} \neq 0$. Remembering that $X \sim \operatorname{det}\left(\bar{q}_{s R} q_{t L}\right)$, up to a multiplicative constant, we find that $F_{X}$ is proportional to the new $2 L$-fermion condensate $C_{U(1)}=\left\langle\mathcal{O}_{U(1)}\right\rangle$ introduced above. In the same way, the pion decay constant $F_{\pi}$, which controls the breaking of the $S U(L) \otimes S U(L)$ symmetry, is related to the $q \bar{q}$ chiral condensate by a simple and well-known proportionality relation (see Refs. [6, 10] and references therein): $\left\langle\bar{q}_{i} q_{i}\right\rangle_{T<T_{c h}} \simeq-\frac{1}{2} B_{m} F_{\pi}$. (Moreover, in the simple case of $L$ light quarks with the same mass $m, m_{N S}^{2}=m B_{m} / F_{\pi}$ is the squared mass of the non-singlet pseudoscalar mesons and one gets the well-known Gell-MannOakes-Renner relation: $m_{N S}^{2} F_{\pi}^{2} \simeq-2 m\left\langle\bar{q}_{i} q_{i}\right\rangle_{T<T_{c h}}$.) It is not possible to find, in a simple way, the analogous relation between $F_{X}$ and the new condensate $C_{U(1)}=\left\langle\mathcal{O}_{U(1)}\right\rangle$.

However, as was shown in two previous papers [11, 12], information on the quantity $F_{X}$ (i.e., on the new $U(1)$ chiral condensate, to which it is related) can be derived, in the realistic case of $L=3$ light quarks with non-zero masses $m_{u}, m_{d}$ and $m_{s}$, from the study of the radiative decays of the pseudoscalar mesons $\eta$ and $\eta^{\prime}$ into two photons. A first comparison of the results with the experimental data has been performed and it is encouraging, pointing towards some evidence for a non-zero $U(1)$ axial condensate. The following decay rates are derived [11, 12]:

$$
\begin{aligned}
\Gamma(\eta \rightarrow \gamma \gamma) & =\frac{\alpha^{2} m_{\eta}^{3}}{192 \pi^{3} F_{\pi}^{2}}\left(\cos \tilde{\varphi}+\frac{2 \sqrt{2} F_{\pi}}{F_{\eta^{\prime}}} \sin \tilde{\varphi}\right)^{2}, \\
\Gamma\left(\eta^{\prime} \rightarrow \gamma \gamma\right) & =\frac{\alpha^{2} m_{\eta^{\prime}}^{3}}{192 \pi^{3} F_{\pi}^{2}}\left(\frac{2 \sqrt{2} F_{\pi}}{F_{\eta^{\prime}}} \cos \tilde{\varphi}-\sin \tilde{\varphi}\right)^{2},
\end{aligned}
$$

where $\alpha=e^{2} / 4 \pi \simeq 1 / 137$ is the fine-structure constant. Here $F_{\eta^{\prime}}$ is defined as follows:

$$
F_{\eta^{\prime}} \equiv \sqrt{F_{\pi}^{2}+3 F_{X}^{2}}
$$

and can be identified with the $\eta^{\prime}$ decay constant in the chiral limit of zero quark masses. Moreover, $\tilde{\varphi}$ is a mixing angle, which can be related to the masses of the quarks $m_{u}, m_{d}$, $m_{s}$, and therefore to the masses of the octet mesons, by the following relation:

$$
\tan \tilde{\varphi}=\frac{\sqrt{2}}{9 A} B F_{\pi} F_{\eta^{\prime}}\left(m_{s}-\tilde{m}\right)=\frac{F_{\pi} F_{\eta^{\prime}}}{6 \sqrt{2} A}\left(m_{\eta}^{2}-m_{\pi}^{2}\right),
$$

where: $m_{\pi}^{2}=2 B \tilde{m}$ and $m_{\eta}^{2}=\frac{2}{3} B\left(\tilde{m}+2 m_{s}\right)$, with: $B \equiv \frac{B_{m}}{2 F_{\pi}}$ and $\tilde{m} \equiv \frac{m_{u}+m_{d}}{2}$. If one puts $F_{X}=0$, i.e., if one neglects the new $U(1)$ chiral condensate, the expressions 
written above reduce to the corresponding ones derived in Ref. [23] using an effective Lagrangian which includes only the usual $q \bar{q}$ chiral condensate. Using the experimental values for the various quantities which appear in Eqs. (1.5) and (1.6), one can extract the following values for the quantity $F_{X}$ and for the mixing angle $\tilde{\varphi}+\oplus$

$$
F_{X}=24(7) \mathrm{MeV}, \quad \tilde{\varphi}=17(2)^{\circ},
$$

and these values are perfectly consistent with the relation (1.8) for the mixing angle, if one uses for the pure-YM topological susceptibility the estimate $A=(180 \pm 5 \mathrm{MeV})^{4}$, obtained from lattice simulations [25].

In the section 3 of this paper, continuing the work started in Refs. [11, 12, we shall study the effects of the $U(1)$ chiral condensate on the strong decays of the "light" pseudoscalar mesons, i.e., $\eta, \eta^{\prime} \rightarrow 3 \pi^{0} ; \eta, \eta^{\prime} \rightarrow \pi^{+} \pi^{-} \pi^{0} ; \eta^{\prime} \rightarrow \eta \pi^{0} \pi^{0} ; \eta^{\prime} \rightarrow \eta \pi^{+} \pi^{-}$; and also on the strong decays of an exotic ("heavy") $S U(3)$-singlet pseudoscalar state $\eta_{X}$, predicted by the model: $\eta_{X} \rightarrow 3 \pi^{0} ; \eta_{X} \rightarrow \pi^{+} \pi^{-} \pi^{0} ; \eta_{X} \rightarrow \eta \pi^{0} \pi^{0} ; \eta_{X} \rightarrow \eta \pi^{+} \pi^{-}$; $\eta_{X} \rightarrow \eta^{\prime} \pi^{0} \pi^{0} ; \eta_{X} \rightarrow \eta^{\prime} \pi^{+} \pi^{-} ; \eta_{X} \rightarrow 3 \eta, 3 \eta^{\prime}, \eta \eta \eta^{\prime}, \eta \eta^{\prime} \eta^{\prime}$. In particular, in the case of the exotic particle $\eta_{X}$, we shall find some relations between its mass and its decay widths, which in principle might be useful to identify a possible candidate for this particle.

For the benefit of the reader, we shall start, in section 2, by resuming the main results, obtained in the original papers [6, 8, 10, concerning the mass spectrum of the Chiral Effective Lagrangian (1.2)-(1.3), for temperatures $T<T_{c h}$ : in this paper we shall consider the case $T=0$ only.

\footnotetext{
${ }^{\dagger}$ Indeed, the original values reported in Refs. [11, 12] were: $F_{X}=27(9) \mathrm{MeV}$ and $\tilde{\varphi}=16(3)^{\circ}$. The values reported in Eq. (1.9) (which are, anyhow, consistent with the original values within the errors) have been obtained using the updated experimental values of the Particle Data Group 24] (in particular: $\Gamma_{\exp }(\eta \rightarrow \gamma \gamma)=0.51(3) \mathrm{keV}$ and $\Gamma_{\exp }\left(\eta^{\prime} \rightarrow \gamma \gamma\right)=4.31(36) \mathrm{keV}$; moreover we use: $F_{\pi}=92.2(4) \mathrm{MeV}$, $\left.m_{\pi} \simeq 134.98 \mathrm{MeV}, m_{\eta} \simeq 547.85 \mathrm{MeV}, m_{\eta^{\prime}} \simeq 957.78 \mathrm{MeV}\right)$.
} 


\section{Mass spectrum and new parameters of the Chiral Effective Lagrangian}

Let us consider the Lagrangian (1.2), where the field variable $Q(x)$ has been integrated out:

$$
\begin{gathered}
\mathcal{L}\left(U, U^{\dagger}, X, X^{\dagger}\right)=\frac{1}{2} \operatorname{Tr}\left(\partial_{\mu} U \partial^{\mu} U^{\dagger}\right)+\frac{1}{2} \partial_{\mu} X \partial^{\mu} X^{\dagger}-V\left(U, U^{\dagger}, X, X^{\dagger}\right) \\
+\frac{1}{8} A\left[w_{1} \operatorname{Tr}\left(\ln U-\ln U^{\dagger}\right)+\left(1-w_{1}\right)\left(\ln X-\ln X^{\dagger}\right)\right]^{2} .
\end{gathered}
$$

\subsection{Mass spectrum at $T=0$ for a generic $L$ (in the chiral limit)}

At $T=0$ both $S U(L) \otimes S U(L)$ and $U(1)_{A}$ symmetries are broken.

Following Ref. [18, we can eliminate the redundant (having much larger masses) scalar fields of the linear $\sigma$-type model by taking the limit $\lambda_{\pi}^{2} \rightarrow \infty$ and $\lambda_{X}^{2} \rightarrow \infty$. In this limit the potential term gives the following constraints:

$$
U^{\dagger} U=\frac{1}{2} F_{\pi}^{2} \cdot \mathbf{I} ; \quad X^{\dagger} X=\frac{1}{2} F_{X}^{2}
$$

We are thus left with a non-linear chiral effective model, in which the field $U$ has the form:

$$
U=\sqrt{\frac{1}{2}} F_{\pi} \exp \left\{\frac{i \sqrt{2}}{F_{\pi}} \Phi\right\}, \quad \Phi=\sum_{a=1}^{L^{2}-1} \pi_{a} \tau_{a}+\frac{S_{\pi}}{\sqrt{L}} \mathbf{I},
$$

where $\tau_{a}\left(a=1, \cdots, L^{2}-1\right)$ are the generators of $S U(L)\left(\operatorname{Tr}\left(\tau_{a}\right)=0\right)$ in the fundamental representation, with normalization $\operatorname{Tr}\left(\tau_{a} \tau_{b}\right)=\delta_{a b}$, and $\pi_{a}\left(a=1, \cdots, L^{2}-1\right)$ are the non-singlet meson fields, while $S_{\pi}$ is the usual quark-antiquark $S U(L)$ singlet field:

$$
S_{\pi} \sim i \sum_{i=1}^{L}\left(\bar{q}_{i L} q_{i R}-\bar{q}_{i R} q_{i L}\right)
$$

And similarly the field $X$ has the form:

$$
X=\sqrt{\frac{1}{2}} F_{X} \exp \left\{\frac{i \sqrt{2}}{F_{X}} S_{X}\right\}
$$


where $S_{X}$ is an exotic singlet field, with the following quark content:

$$
S_{X} \sim i\left[\operatorname{det}_{s t}\left(\bar{q}_{s L} q_{t R}\right)-\operatorname{det}_{s t}\left(\bar{q}_{s R} q_{t L}\right)\right] .
$$

Substituting Eqs. (2.3) and (2.5) into Eq. (2.1) and taking only the quadratic part of the Lagrangian, we obtain:

$$
\begin{aligned}
\mathcal{L}_{2} & =\frac{1}{2} \partial_{\mu} \pi_{a} \partial^{\mu} \pi_{a}+\frac{1}{2} \partial_{\mu} S_{\pi} \partial^{\mu} S_{\pi}+\frac{1}{2} \partial_{\mu} S_{X} \partial^{\mu} S_{X}-\frac{1}{2}\left(\sum_{i l} \mu_{i}^{2} \tau_{i l}^{a} \tau_{l i}^{b}\right) \pi_{a} \pi_{b} \\
& -\frac{1}{2}\left(\frac{2}{\sqrt{L}} \sum_{i} \mu_{i}^{2} \tau_{i i}^{a}\right) \pi_{a} S_{\pi}-\frac{1}{2 L} \sum_{i} \mu_{i}^{2} S_{\pi}^{2} \\
& -\frac{1}{2} c\left(\frac{\sqrt{2 L}}{F_{\pi}} S_{\pi}-\frac{\sqrt{2}}{F_{X}} S_{X}\right)^{2}-\frac{1}{2} A\left[\frac{\sqrt{2 L}}{F_{\pi}} \omega_{1} S_{\pi}+\frac{\sqrt{2}}{F_{X}}\left(1-\omega_{1}\right) S_{X}\right]^{2},
\end{aligned}
$$

where:

$$
c \equiv \frac{c_{1}}{\sqrt{2}}\left(\frac{F_{X}}{\sqrt{2}}\right)\left(\frac{F_{\pi}}{\sqrt{2}}\right)^{L}, \quad \mu_{i}^{2} \equiv \frac{B_{m}}{F_{\pi}} m_{i} .
$$

In the chiral limit, $\sup m_{i} \rightarrow 0$, Eq. (2.7) reduces to:

$$
\begin{aligned}
\mathcal{L}_{2} & =\frac{1}{2} \partial_{\mu} \pi_{a} \partial^{\mu} \pi_{a}+\frac{1}{2} \partial_{\mu} S_{\pi} \partial^{\mu} S_{\pi}+\frac{1}{2} \partial_{\mu} S_{X} \partial^{\mu} S_{X} \\
& -\frac{1}{2} c\left(\frac{\sqrt{2 L}}{F_{\pi}} S_{\pi}-\frac{\sqrt{2}}{F_{X}} S_{X}\right)^{2}-\frac{1}{2} A\left[\frac{\sqrt{2 L}}{F_{\pi}} \omega_{1} S_{\pi}+\frac{\sqrt{2}}{F_{X}}\left(1-\omega_{1}\right) S_{X}\right]^{2} .
\end{aligned}
$$

In this case the $L^{2}-1$ non-singlet fields are massless: they are the Goldstone bosons coming from the breaking of the $S U(L) \otimes S U(L)$ symmetry down to $S U(L)_{V}$. Instead, the two singlet fields $S_{\pi}$ and $S_{X}$ are mixed with the following squared mass matrix:

$$
\left(\begin{array}{cc}
\frac{2 L\left(A \omega_{1}^{2}+c\right)}{F_{\pi}^{2}} & \frac{2 \sqrt{L}\left[A \omega_{1}\left(1-\omega_{1}\right)-c\right]}{F_{\pi} F_{X}} \\
\frac{2 \sqrt{L}\left[A \omega_{1}\left(1-\omega_{1}\right)-c\right]}{F_{\pi} F_{X}} & \frac{2\left[A\left(1-\omega_{1}\right)^{2}+c\right]}{F_{X}^{2}}
\end{array}\right) .
$$

The eigenvalues of this matrix are:

$$
m_{S_{1}, S_{2}}^{2}=\frac{Z_{L} \mp \sqrt{Z_{L}^{2}-4 Q_{L}}}{2},
$$


where:

$$
\begin{aligned}
Z_{L} & \equiv \frac{2 A\left[F_{\pi}^{2}\left(1-\omega_{1}\right)^{2}+L F_{X}^{2} \omega_{1}^{2}\right]+2 c\left(F_{\pi}^{2}+L F_{X}^{2}\right)}{F_{\pi}^{2} F_{X}^{2}}, \\
Q_{L} & \equiv \frac{4 L A c}{F_{\pi}^{2} F_{X}^{2}} .
\end{aligned}
$$

Making use of the following $N$-dependences of the relevant quantities in the limit of large number of colours $N$ (see Ref. [6]):

$$
F_{\pi}=\mathcal{O}\left(N^{1 / 2}\right) ; \quad F_{X}=\mathcal{O}\left(N^{1 / 2}\right) ; \quad A=\mathcal{O}(1) ; \quad c=\mathcal{O}(N),
$$

we derive, at the first order in the $1 / N$ expansion (and assuming that $c_{1} \neq 0$ : see the discussion in Appendix B), the following expressions for the two eigenvectors:

$$
\begin{aligned}
& S_{1}=\frac{1}{\sqrt{F_{\pi}^{2}+L F_{X}^{2}}}\left(F_{\pi} S_{\pi}+\sqrt{L} F_{X} S_{X}\right), \\
& S_{2}=\frac{1}{\sqrt{F_{\pi}^{2}+L F_{X}^{2}}}\left(\sqrt{L} F_{X} S_{\pi}-F_{\pi} S_{X}\right),
\end{aligned}
$$

with the corresponding eigenvalues:

$$
\begin{aligned}
m_{S_{1}}^{2} & =\frac{2 L A}{F_{\pi}^{2}+L F_{X}^{2}}=\mathcal{O}(1 / N), \\
m_{S_{2}}^{2} & =\frac{2 c\left(F_{\pi}^{2}+L F_{X}^{2}\right)}{F_{\pi}^{2} F_{X}^{2}}=\mathcal{O}(1) .
\end{aligned}
$$

The two fields $S_{1}$ and $S_{2}$ have the same quantum numbers, but different quark contents: the first one (assuming that $F_{\pi} \gg F_{X}$ ) is prevalently a quark-antiquark singlet $S_{\pi}$, while the second one is prevalently an exotic $2 L$-fermion singlet $S_{X} \sim i\left[\operatorname{det}\left(\bar{q}_{s L} q_{t R}\right)-\right.$ $\left.\operatorname{det}\left(\bar{q}_{s R} q_{t L}\right)\right]$. Both fields are massive in the chiral limit. If we let $F_{X} \rightarrow 0$ in the abovereported formulae (i.e., if we neglect the new $U(1)$ axial condensate), then $S_{1} \rightarrow S_{\pi}$ and $m_{S_{1}}^{2} \rightarrow 2 L A / F_{\pi}^{2}$, which is the usual Witten-Veneziano formula for the $\eta^{\prime}$ mass in the chiral limit [4, 5]. On the other side, $m_{S_{2}}^{2} \simeq 2 c / F_{X}^{2} \rightarrow \infty$ for $F_{X} \rightarrow 0$, being $c=\mathcal{O}\left(F_{X}\right)$ [Eq. (2.8)], and therefore, in this limit, the field $S_{2} \rightarrow-S_{X}$ is "constrained" to be zero*

${ }^{*}$ More rigorously, before taking the limit $F_{X} \rightarrow 0$ (i.e., $X \rightarrow 0$ ), one should first take the limit $\omega_{1} \rightarrow 1$, so that no singular behaviour arises from the anomalous term in Eqs. (1.2) and (2.1) and the Lagrangian simply reduces, for $X \rightarrow 0$, to the usual Lagrangian of Witten, Di Vecchia, Veneziano et al. It is easy to check that, by putting $\omega_{1}=1$ in Eqs. (2.11)-2.12) and then letting $F_{X} \rightarrow 0$, one recovers the same results that one also obtains by simply letting $F_{X} \rightarrow 0$ in Eqs. (2.15), i.e., $m_{S_{1}}^{2} \rightarrow 2 L A / F_{\pi}^{2}$ and $m_{S_{2}}^{2} \simeq 2 c / F_{X}^{2} \rightarrow \infty$ 
In the more general case $F_{X} \neq 0$, which we are considering in this paper, there is a field $\left(S_{1}\right)$ with a squared mass which vanishes as $\mathcal{O}(1 / N)$ in the large $-N$ expansion; on the contrary, the field $S_{2}$ has a large mass of order $\mathcal{O}(1)$ in the large $-N$ limit. It is quite easy to convince oneself that the particle associated with the field $S_{1}$ is nothing but the particle $\eta^{\prime}$, which is required by the well-known Witten-Veneziano mechanism for the solution of the $U(1)$ problem (see Refs. [8, [10]). In fact, the expression for the $U(1)$ axial current:

$$
\begin{aligned}
J_{5, \mu}^{(L)} & =i\left[\operatorname{Tr}\left(U^{\dagger} \partial_{\mu} U-U \partial_{\mu} U^{\dagger}\right)+L\left(X^{\dagger} \partial_{\mu} X-X \partial_{\mu} X^{\dagger}\right)\right] \\
& =-\sqrt{2 L} \partial_{\mu}\left(F_{\pi} S_{\pi}+\sqrt{L} F_{X} S_{X}\right)
\end{aligned}
$$

can be re-written, using the first Eq. (2.14), as:

$$
J_{5, \mu}^{(L)}=-\sqrt{2 L} F_{S_{1}} \partial_{\mu} S_{1}
$$

where :

$$
F_{S_{1}}=\sqrt{F_{\pi}^{2}+L F_{X}^{2}}
$$

is nothing but the decay constant of the singlet meson $S_{1}$, defined as:

$$
\left\langle 0\left|J_{5, \mu}^{(L)}(0)\right| S_{1}\left(\vec{p}_{1}\right)\right\rangle=i \sqrt{2 L} F_{S_{1}} p_{1 \mu}
$$

We remind that, according to the Witten-Veneziano mechanism for the solution of the $U(1)$ problem, the $\eta^{\prime}$ mass must satisfy the following relation, known as the WittenVeneziano formula:

$$
m_{\eta^{\prime}}^{2}=\frac{2 L A}{F_{\eta^{\prime}}^{2}} .
$$

Using the first Eq. (2.15), together with Eq. (2.18), one immediately verifies that the singlet meson associated with the field $S_{1}$ indeed verifies this relation, i.e., $m_{S_{1}}^{2}=2 L A / F_{S_{1}}^{2}$. For this reason, from now on, the field/particle $S_{1}$ will be denoted as $\eta^{\prime}$, with:

$$
F_{\eta^{\prime}} \equiv F_{S_{1}}=\sqrt{F_{\pi}^{2}+L F_{X}^{2}}
$$

Instead, from now on, we shall use the name $\eta_{X}$ to denote the other exotic singlet field/particle $S_{2}$. 


\subsection{Mass spectrum at $T=0$ for the realistic $L=3$ case}

Let us consider more carefully the realistic case [8], in which there are $L=3$ light quark flavours, named $u, d$ and $s$, with masses $m_{u}=(1.7 \div 3.3) \mathrm{MeV}, m_{d}=(4.1 \div 5.8) \mathrm{MeV}$ and $m_{s}=(80 \div 130) \mathrm{MeV}$ [24], which are small compared to the QCD mass-scale $\Lambda_{Q C D} \sim$ $0.5 \mathrm{GeV}$. In this case Eq. (2.3) becomes:

$$
U=\sqrt{\frac{1}{2}} F_{\pi} \exp \left\{\frac{i \sqrt{2}}{F_{\pi}} \Phi\right\}, \quad \Phi=\sum_{a=1}^{8} \pi_{a} \tau_{a}+\frac{S_{\pi}}{\sqrt{3}} \mathbf{I}
$$

where $\pi_{a}(a=1, \cdots, 8)$ are the pseudoscalar mesons $\left(J^{P}=0^{-}\right)$of the octet, while $S_{\pi}$ is the quark-antiquark $S U(3)$-singlet field. Proceeding as in the previous section, but making also an expansion up to the first order in the quark masses, we immediately find that the fields $\pi_{1}, \pi_{2}, \pi_{4}, \pi_{5}, \pi_{6}, \pi_{7}$ are already diagonal, with masses:

$$
\begin{aligned}
& m_{\pi_{1,2}}^{2} \equiv m_{\pi^{ \pm}}^{2}=B\left(m_{u}+m_{d}\right), \\
& m_{\pi_{4,5}}^{2} \equiv m_{K^{ \pm}}^{2}=B\left(m_{u}+m_{s}\right), \\
& m_{\pi_{6,7}}^{2} \equiv m_{K^{0}, \bar{K}^{0}}^{2}=B\left(m_{d}+m_{s}\right),
\end{aligned}
$$

where $B \equiv \frac{B_{m}}{2 F_{\pi}}$.

On the contrary the fields $\pi_{3}, \pi_{8}, S_{\pi}, S_{X}$ mix together, with the following squared mass matrix:

$$
\mathcal{K}=\left(\begin{array}{cccc}
2 B \tilde{m} & \frac{1}{\sqrt{3}} B \Delta & \sqrt{\frac{2}{3}} B \Delta & 0 \\
\frac{1}{\sqrt{3}} B \Delta & \frac{2}{3} B\left(\tilde{m}+2 m_{s}\right) & \frac{2 \sqrt{2}}{3} B\left(\tilde{m}-m_{s}\right) & 0 \\
\sqrt{\frac{2}{3}} B \Delta & \frac{2 \sqrt{2}}{3} B\left(\tilde{m}-m_{s}\right) & \frac{6\left(A \omega_{1}^{2}+c\right)}{F_{\pi}^{2}}+m_{0}^{2} & \frac{2 \sqrt{3}\left[A\left(1-\omega_{1}\right) \omega_{1}-c\right]}{F_{\pi} F_{X}} \\
0 & 0 & \frac{2 \sqrt{3}\left[\left(1-\omega_{1}\right) \omega_{1}-c\right]}{F_{\pi} F_{X}} & \frac{2\left[A\left(1-\omega_{1}\right)^{2}+c\right]}{F_{X}^{2}}
\end{array}\right)
$$

where $\tilde{m} \equiv \frac{m_{u}+m_{d}}{2}, m_{0}^{2} \equiv \frac{2}{3} B\left(2 \tilde{m}+m_{s}\right)$ and $\Delta \equiv m_{u}-m_{d}$. This last parameter $\Delta$ measures isospin violations, i.e., the explicit breaking of the $S U(2)_{V}$ symmetry. If we neglect the experimentally small violations of the $S U(2)_{V}$ isospin symmetry, i.e., if we 
put $\Delta=0$ in Eq. (2.24) $)^{\dagger}$, the squared mass matrix (2.24) simplifies to:

$$
\mathcal{K}_{0}=\left(\begin{array}{cccc}
2 B \tilde{m} & 0 & 0 & 0 \\
0 & \frac{2}{3} B\left(\tilde{m}+2 m_{s}\right) & \frac{2 \sqrt{2}}{3} B\left(\tilde{m}-m_{s}\right) & 0 \\
0 & \frac{2 \sqrt{2}}{3} B\left(\tilde{m}-m_{s}\right) & \frac{6\left(A \omega_{1}^{2}+c\right)}{F_{\pi}^{2}}+m_{0}^{2} & \frac{2 \sqrt{3}\left[A\left(1-\omega_{1}\right) \omega_{1}-c\right]}{F_{\pi} F_{X}} \\
0 & 0 & \frac{2 \sqrt{3}\left[A\left(1-\omega_{1}\right) \omega_{1}-c\right]}{F_{\pi} F_{X}} & \frac{2\left[A\left(1-\omega_{1}\right)^{2}+c\right]}{F_{X}^{2}}
\end{array}\right)
$$

Therefore, in this limit, $\pi_{3}$ also becomes diagonal and can be identified with the physical state $\pi^{0}$, with squared mass:

$$
m_{\pi^{0}}^{2}=2 B \tilde{m}=B\left(m_{u}+m_{d}\right) \equiv m_{\pi}^{2} .
$$

The fields $\left(\pi_{3}, \pi_{8}, S_{\pi}, S_{X}\right)$ can be written in terms of the eigenstates $\left(\pi^{0}, \eta, \eta^{\prime}, \eta_{X}\right)$ as follows:

$$
\left(\begin{array}{c}
\pi_{3} \\
\pi_{8} \\
S_{\pi} \\
S_{X}
\end{array}\right)=\mathbf{C}_{0}\left(\begin{array}{c}
\pi^{0} \\
\eta \\
\eta^{\prime} \\
\eta_{X}
\end{array}\right)
$$

where $\mathbf{C}_{0}$ is the following orthogonal matrix [11, 12]:

$$
\mathbf{C}_{0}=\left(\begin{array}{cccc}
1 & 0 & 0 & 0 \\
0 & \cos \tilde{\varphi} & -\sin \tilde{\varphi} & 0 \\
0 & \frac{F_{\pi}}{F_{\eta^{\prime}}} \sin \tilde{\varphi} & \frac{F_{\pi}}{F_{\eta^{\prime}}} \cos \tilde{\varphi} & \frac{\sqrt{3} F_{X}}{F_{\eta^{\prime}}} \\
0 & \frac{\sqrt{3} F_{X}}{F_{\eta^{\prime}}} \sin \tilde{\varphi} & \frac{\sqrt{3} F_{X}}{F_{\eta^{\prime}}} \cos \tilde{\varphi} & -\frac{F_{\pi}}{F_{\eta^{\prime}}}
\end{array}\right)
$$

As we have already said above, $F_{\eta^{\prime}} \equiv \sqrt{F_{\pi}^{2}+3 F_{X}^{2}}$ can be identified with the $\eta^{\prime}$ decay constant in the chiral limit of zero quark masses [11, 12]. Moreover, $\tilde{\varphi}$ is a mixing angle, which can be related to the masses of the quarks $m_{u}, m_{d}, m_{s}$, and therefore to the masses of the octet mesons, by the relation (1.8) [11, 12].

The matrix $\mathbf{C}_{0}$ has been derived by diagonalizing the squared mass matrix (2.25) at the first order in the quark masses and in $1 / N$, so neglecting terms behaving as $1 / N^{2}$, $m^{2}$ or $m / N$ (and assuming, again, that $c_{1} \neq 0$ : see the discussion in Appendix B).

\footnotetext{
${ }^{\dagger}$ In the next section, instead, we shall take into account also the small violations of the $S U(2)_{V}$ isospin symmetry, by taking $\Delta \neq 0$.
} 
Following Refs. [5, 18, 23], we have considered the limit in which $m / \Lambda_{Q C D} \ll 1 / N \ll 1$ : this particular choice is justified by the fact that the mixing angle, which is of order $\mathcal{O}\left(m N / \Lambda_{Q C D}\right)$, is experimentally smalt ${ }^{\ddagger}$. The other eigenvalues of the squared mass matrix (2.25) can be easily derived at the first order in the quark masses and in $1 / N$ (in the sense explained above):

$$
\begin{aligned}
m_{\eta}^{2} & =\frac{2}{3} B\left(\tilde{m}+2 m_{s}\right) \\
m_{\eta^{\prime}}^{2} & =\frac{6 A}{F_{\eta^{\prime}}^{2}}+\frac{F_{\pi}^{2}}{F_{\eta^{\prime}}^{2}} m_{0}^{2} \\
m_{\eta_{X}}^{2} & =\frac{2 c F_{\eta^{\prime}}^{2}}{F_{\pi}^{2} F_{X}^{2}}+\frac{2 A\left[F_{\pi}^{2}\left(\omega_{1}-1\right)+3 F_{X}^{2} \omega_{1}\right]^{2}}{F_{\pi}^{2} F_{X}^{2} F_{\eta^{\prime}}^{2}}+\frac{3 F_{X}^{2}}{F_{\eta^{\prime}}^{2}} m_{0}^{2}
\end{aligned}
$$

The physical interpretation of these three states is clear. The state $\eta$ is the eighth pseudoGoldstone bosons of the octet: its mass vanishes with the light quark masses. On the contrary, the states $\eta^{\prime}$ and $\eta_{X}$ have masses which do not vanish with the light quark masses. In particular, the state $\eta^{\prime}$ has a topological (non-chiral) squared mass term $6 A / F_{\eta^{\prime}}^{2}$, which vanishes as $1 / N$ in the large $-N$ limit. The state $\eta_{X}$, instead, should be heavier, having a normal (non chiral) mesonic mass term $\S$ of order $\mathcal{O}(1)$ in the large $-N$ limit.

From Eqs. (2.23), (2.26) and (2.29) one immediately derives the well-known Gell-MannOkubo formula [28, 29] for the squared masses of the octet mesons:

$$
3 m_{\eta}^{2}+m_{\pi}^{2}=4 m_{K}^{2}
$$

where: $m_{K}^{2} \equiv \frac{1}{2}\left(m_{K^{ \pm}}^{2}+m_{K^{0}, \bar{K}^{0}}^{2}\right)=B\left(\tilde{m}+m_{s}\right)$. In fact, it is natural to expect that the introduction of a new chiral order parameter, which only breaks the $U(1)$ axial symmetry, should not modify the mass relations for the octet mesons, such as Eq. (2.32), which only derive from the breaking of $S U(3) \otimes S U(3)$ down to $S U(3)_{V}$.

Considering also the squared mass (2.30) of the $\eta^{\prime}$, one immediately derives the following interesting relation (with $m_{K}^{2}$ defined as in Eq. (2.32)) [8]:

$$
\left(1+3 \frac{F_{X}^{2}}{F_{\pi}^{2}}\right) m_{\eta^{\prime}}^{2}+m_{\eta}^{2}-2 m_{K}^{2}=\frac{6 A}{F_{\pi}^{2}} .
$$

\footnotetext{
${ }^{\ddagger}$ In the literature, also other possibilities have been studied. For example, Leutwyler in Ref. [26] considers $m / \Lambda_{Q C D}$ and $1 / N$ to be of the same order, and Witten in Ref. [19] studies also the opposite case, i.e., $m N / \Lambda_{Q C D} \gg 1$.

${ }^{\S}$ See Ref. [27] for a detailed discussion of hadrons and their masses in the framework of the $1 / N$ expansion.
} 
This is nothing but a generalization of the usual Witten-Veneziano formula for the $\eta^{\prime}$ mass (including non-zero quark masses), with a correction which only depends on the parameter $F_{X}$ (which, as we have already said in the Introduction, is essentially proportional to the new $U(1)$ axial condensate), but not on the other unknown parameters of the model $\left(\omega_{1}\right.$, $c_{1}$ ). From Eq. (2.33), using the known values for the meson masses, the pion decay constant $F_{\pi}$ and the pure-gauge topological susceptibility $A$, one can derive the following upper limit for the parameter $F_{X}:\left|F_{X}\right| \lesssim 20 \mathrm{MeV}$ [8, 10].

Finally, we can derive an anologous relation involving also the squared mass of the exotic state $\eta_{X}$. By taking the trace of the squared mass matrix (2.24), using the relations (2.23), together with $\tilde{m} \equiv \frac{m_{u}+m_{d}}{2}, m_{0}^{2} \equiv \frac{2}{3} B\left(2 \tilde{m}+m_{s}\right)$ and $m_{K}^{2} \equiv B\left(\tilde{m}+m_{s}\right)$, one obtains:

$$
\begin{aligned}
\operatorname{Tr}[\mathcal{K}] & =m_{\pi^{0}}^{2}+m_{\eta}^{2}+m_{\eta^{\prime}}^{2}+m_{\eta_{X}}^{2} \\
& =2 B \tilde{m}+\frac{2}{3} B\left(\tilde{m}+2 m_{s}\right)+m_{0}^{2}+\frac{6\left(A \omega_{1}^{2}+c\right)}{F_{\pi}^{2}}+\frac{2 A\left(1-\omega_{1}\right)^{2}+2 c}{F_{X}^{2}} \\
& =m_{\pi^{0}}^{2}+2 m_{K}^{2}+\frac{6\left(A \omega_{1}^{2}+c\right)}{F_{\pi}^{2}}+\frac{2 A\left(1-\omega_{1}\right)^{2}+2 c}{F_{X}^{2}},
\end{aligned}
$$

from which, re-ordering, one finally gets:

$$
m_{\eta_{X}}^{2}+m_{\eta^{\prime}}^{2}+m_{\eta}^{2}-2 m_{K}^{2}=\frac{2 c F_{\eta^{\prime}}^{2}}{F_{\pi}^{2} F_{X}^{2}}+\frac{2 A\left[F_{\pi}^{2}\left(1-\omega_{1}\right)^{2}+3 F_{X}^{2} \omega_{1}^{2}\right]}{F_{\pi}^{2} F_{X}^{2}}
$$

Unfortunately, this expression depends upon all the unknown parameters of the model $\left(F_{X}, \omega_{1}, c_{1}\right)$ and, therefore, we cannot use it to obtain a direct estimate of the mass of the particle $\eta_{X}$. However, in the next section we shall find some relations between its mass and its decay widths, which in principle might be useful to identify a possible candidate for this particle. 


\section{The strong decays of the pseudoscalar mesons $\eta, \eta^{\prime}, \eta_{X}$}

In this section we shall study the strong decays of pseudoscalar mesons, using the Chiral Effective Lagrangian which we have discussed above.

First we observe that the strong decays of a pseudoscalar meson into two pseudoscalar mesons are trivially forbidden by parity conservation. In fact, in terms of the Chiral Effective Lagrangian (2.1), one easily verifies that it is invariant under the following field transformation:

$$
U \rightarrow U^{\dagger}, \quad X \rightarrow X^{\dagger}, \quad Q \rightarrow-Q,
$$

which is nothing but the parity transformation for the fields [provided one also transforms the space-time coordinates as $\left.x=\left(x^{0}, \vec{x}\right) \rightarrow x_{P}=\left(x^{0},-\vec{x}\right)\right]$. In terms of the meson fields $\pi_{a}, S_{\pi}, S_{X}$, defined in Eqs. (2.3) and (2.5), it corresponds to:

$$
\pi_{a} \rightarrow-\pi_{a}, \quad S_{\pi} \rightarrow-S_{\pi}, \quad S_{X} \rightarrow-S_{X} .
$$

Therefore, terms with an odd number of meson fields (which are not parity invariant) necessarily vanish. In particular, operators with three pseudoscalar meson fields are absent and therefore the strong decays of a pseudoscalar meson into two pseudoscalar mesons are forbidden.

On the contrary, the strong decays of a pseudoscalar meson into three pseudoscalar mesons, being induced by parity-invariant four-meson operators, are allowed and we shall devote the rest of this section to a detailed discussions of these decays.

\subsection{The four-meson Lagrangian}

In order to study the strong decays of $\eta, \eta^{\prime}, \eta_{X}$ into three pseudoscalar mesons, we have to isolate the four-meson operators in the Lagrangian (2.1), when expanding the fields (2.3) and (2.5) in powers of the meson fields. We thus obtain the following four-meson Lagrangian:

$$
\begin{aligned}
\mathcal{L}_{4} & =\frac{1}{4 F_{\pi}^{2}} \operatorname{Tr}\left[\partial_{\mu} \Phi^{2} \partial^{\mu} \Phi^{2}+\frac{4}{3} \Phi^{3} \square \Phi\right]+\frac{1}{4 F_{X}^{2}}\left[\partial_{\mu} S_{X}^{2} \partial^{\mu} S_{X}^{2}+\frac{4}{3} S_{X}^{3} \square S_{X}\right] \\
& +\frac{B}{6 F_{\pi}^{2}} \operatorname{Tr}\left[M \Phi^{4}\right]+\frac{c}{6}\left(\frac{\sqrt{3}}{F_{\pi}} S_{\pi}-\frac{1}{F_{X}} S_{X}\right)^{4},
\end{aligned}
$$


where, as usual: $B=\frac{B_{m}}{2 F_{\pi}}, c=\frac{c_{1}}{\sqrt{2}}\left(\frac{F_{X}}{\sqrt{2}}\right)\left(\frac{F_{\pi}}{\sqrt{2}}\right)^{3}$.

By making an integration by parts and using the usual identitites for the $S U(3)$ generators, we can re-write the first term in the r.h.s. of Eq. (3.3) as (apart from total derivatives):

$$
\begin{aligned}
\delta \mathcal{L}_{4}^{(f)} & =\frac{1}{4 F_{\pi}^{2}} \operatorname{Tr}\left[\partial_{\mu} \Phi^{2} \partial^{\mu} \Phi^{2}+\frac{4}{3} \Phi^{3} \square \Phi\right]=\frac{1}{4 F_{\pi}^{2}} \operatorname{Tr}\left[\partial_{\mu} \Phi^{2} \partial^{\mu} \Phi^{2}-\frac{4}{3} \partial_{\mu} \Phi^{3} \partial^{\mu} \Phi\right] \\
& =\frac{1}{4 F_{\pi}^{2}}\left[-\frac{2}{3} f_{i j c} f_{c \alpha \beta}\left(\pi_{i} \partial_{\mu} \pi_{j}\right)\left(\pi_{\alpha} \partial^{\mu} \pi_{\beta}\right)\right]
\end{aligned}
$$

where $f_{a b c}$ are the structure constants of $S U(3)$, defined as: $\left[\tau_{a}, \tau_{b}\right]=i \sqrt{2} f_{a b c} \tau_{c}$, with $\operatorname{Tr}\left(\tau_{a} \tau_{b}\right)=\delta_{a b}$. It is easy to see that this term gives contributions only to decays into charged pions, whose fields are $\pi^{ \pm}=\frac{\pi_{1} \mp i \pi_{2}}{\sqrt{2}}$.

Concerning the second term in the r.h.s. of Eq. (3.3), we immediately recognize (after an integration by parts) that it vanishes (apart from a total derivative):

$$
\frac{1}{4 F_{X}^{2}}\left[\partial_{\mu} S_{X}^{2} \partial^{\mu} S_{X}^{2}+\frac{4}{3} S_{X}^{3} \square S_{X}\right]=\frac{1}{4 F_{X}^{2}}\left[\partial_{\mu} S_{X}^{2} \partial^{\mu} S_{X}^{2}-\frac{4}{3} \partial_{\mu} S_{X}^{3} \partial^{\mu} S_{X}\right]=0
$$

Therefore, the four-meson Lagrangian (3.3) reduces to:

$$
\begin{aligned}
\mathcal{L}_{4} & =\frac{1}{4 F_{\pi}^{2}}\left[-\frac{2}{3} f_{i j c} f_{c \alpha \beta} \pi_{i} \pi_{\alpha} \partial_{\mu} \pi_{j} \partial^{\mu} \pi_{\beta}\right]+\frac{B}{6 F_{\pi}^{2}} \operatorname{Tr}\left[M \Phi^{4}\right] \\
& +\frac{c}{6}\left(\frac{\sqrt{3}}{F_{\pi}} S_{\pi}-\frac{1}{F_{X}} S_{X}\right)^{4} .
\end{aligned}
$$

In the limit $c \rightarrow 0, F_{X} \rightarrow 0$ and $S_{X} \rightarrow 0$ this Lagrangian reduces to the usual four-meson Lagrangian derived by Di Vecchia et al. in Ref. [23].

The last term in the four-meson Lagrangian (3.6) can be re-written in terms of the mass eigenstates, given, in the case $\Delta=0$, by Eqs. (2.27)-(2.28), so obtaining:

$$
\delta \mathcal{L}_{4}^{(c)}=\frac{c}{6}\left(\frac{\sqrt{3}}{F_{\pi}} S_{\pi}-\frac{1}{F_{X}} S_{X}\right)^{4}=\frac{c}{6}\left(\frac{F_{\eta^{\prime}}}{F_{\pi} F_{X}}\right)^{4} \eta_{X}^{4}
$$

This term contributes only to the elastic scattering amplitude $\eta_{X} \eta_{X} \rightarrow \eta_{X} \eta_{X}$. At the end of the next subsection we shall see that, for $\Delta \equiv m_{u}-m_{d} \neq 0$, the term $\delta \mathcal{L}_{4}^{(c)}$ gives also contributions to the decays into three pseudoscalar mesons, but these contributions are strongly suppressed for small $\Delta$. 


\subsection{The mass eigenstates in the case $\Delta \neq 0$}

In the strong decays of $\eta, \eta^{\prime}, \eta_{X}$ into three pions the $S U(2)$ isotopic spin $\hat{\vec{I}}$ is not conserved, i.e. (being the charge conjugation $\hat{C}$ conserved by strong interactions) the so-called $G^{-}$ parity, defined, for a multiplet of isotopic spin $I$, as $\hat{G} \equiv \hat{C} e^{i \pi \hat{I}_{2}}=C_{0}(-1)^{I}, C_{0}$ being the eigenvalue of $\hat{C}$ for the neutral component of the multiplet, is not conserved. The mesons $\eta, \eta^{\prime}, \eta_{X}$ are isosinglets $(I=0)$ with $C=1$ (they can decay into $\gamma \gamma$ for the electromagnetic interaction), and so they have $G=1$. On the contrary, the mesons $\pi$ form an isotriplet $(I=1)$, with $C_{0}=1$ (since $\pi^{0}$ can decay into $\gamma \gamma$ for the electromagnetic interaction), and so each of them has $G=-1$, and a three-pion final state has $G=(-1)^{3}=-1$.

We shall evaluate the decay amplitudes (and the corresponding decay widths) at the lowest order in the parameter $\Delta \equiv m_{u}-m_{d}$, which measures isospin violations, i.e., the explict breaking of the $S U(2)_{V}$ symmetry. In the case $\Delta \neq 0$, the fields $\pi_{3}, \pi_{8}, S_{\pi}, S_{X}$ mix together with the squared mass matrix $\mathcal{K}$, given by Eq. (2.24), while the remaining $\pi_{a}$ are already diagonal [8]. We write the matrix $\mathcal{K}$ as:

$$
\mathcal{K}=\mathcal{K}_{0}+\delta \mathcal{K}_{\Delta}
$$

where $\mathcal{K}_{0}$ is the matrix $\mathcal{K}$ for $\Delta=0$, given by Eq. (2.25), which is diagonalized by the orthogonal matrix $\mathbf{C}_{0}$, given by Eq. (2.28), while $\delta \mathcal{K}_{\Delta}$ is given by:

$$
\delta \mathcal{K}_{\Delta}=\left(\begin{array}{cccc}
0 & \frac{1}{\sqrt{3}} B \Delta & \sqrt{\frac{2}{3}} B \Delta & 0 \\
\frac{1}{\sqrt{3}} B \Delta & 0 & 0 & 0 \\
\sqrt{\frac{2}{3}} B \Delta & 0 & 0 & 0 \\
0 & 0 & 0 & 0
\end{array}\right) .
$$

We shall evaluate the eigenvalues and the eigenstates of the matrix $\mathcal{K}$ at the first order in the parameter $\Delta$, by treating the term $\delta \mathcal{K}_{\Delta}$ as a small perturbation. It is easy to verify that the corrections to the eigenvalues (i.e., to the squared masses $m_{\pi}^{2}, m_{\eta}^{2}, m_{\eta^{\prime}}^{2}$, $m_{\eta_{X}}^{2}$, evaluated in the previous section) are of order $\Delta^{2}$ (the first-order corrections being identically zero) and are therefore negligible, if we stop at the first order in $\Delta$. Instead, the eigenstates of the matrix $\mathcal{K}$ at the first order in the parameter $\Delta$ are given by:

$$
\left(\begin{array}{c}
\pi_{3} \\
\pi_{8} \\
S_{\pi} \\
S_{X}
\end{array}\right)=\mathbf{C}\left(\begin{array}{c}
\pi^{0} \\
\eta \\
\eta^{\prime} \\
\eta_{X}
\end{array}\right), \quad \mathbf{C}=\left(\begin{array}{cccc}
\delta_{0} & \delta_{1} & \delta_{2} & \delta_{3} \\
\alpha_{0} & \alpha_{1} & \alpha_{2} & \alpha_{3} \\
\beta_{0} & \beta_{1} & \beta_{2} & \beta_{3} \\
\gamma_{0} & \gamma_{1} & \gamma_{2} & \gamma_{3}
\end{array}\right)
$$


where:

$$
\begin{aligned}
& \delta_{0}=1 \text {, } \\
& \alpha_{0}=\frac{B \Delta}{\sqrt{3}}\left[\frac{\cos \tilde{\varphi}}{\left(m_{\pi}^{2}-m_{\eta}^{2}\right)}\left(\cos \tilde{\varphi}+\frac{\sqrt{2} F_{\pi}}{F_{\eta^{\prime}}} \sin \tilde{\varphi}\right)\right. \\
& \left.-\frac{\sin \tilde{\varphi}}{\left(m_{\pi}^{2}-m_{\eta^{\prime}}^{2}\right)}\left(\frac{\sqrt{2} F_{\pi}}{F_{\eta^{\prime}}} \cos \tilde{\varphi}-\sin \tilde{\varphi}\right)\right] \text {, } \\
& \beta_{0}=\frac{B \Delta}{\sqrt{3}}\left[\frac{F_{\pi} \sin \tilde{\varphi}}{\left(m_{\pi}^{2}-m_{\eta}^{2}\right) F_{\eta^{\prime}}}\left(\cos \tilde{\varphi}+\frac{\sqrt{2} F_{\pi}}{F_{\eta^{\prime}}} \sin \tilde{\varphi}\right)\right. \\
& \left.+\frac{F_{\pi} \cos \tilde{\varphi}}{\left(m_{\pi}^{2}-m_{\eta^{\prime}}^{2}\right) F_{\eta^{\prime}}}\left(\frac{\sqrt{2} F_{\pi}}{F_{\eta^{\prime}}} \cos \tilde{\varphi}-\sin \tilde{\varphi}\right)+\frac{3 \sqrt{2} F_{X}^{2}}{\left(m_{\pi}^{2}-m_{\eta_{X}}^{2}\right) F_{\eta^{\prime}}^{2}}\right] \text {, } \\
& \gamma_{0}=B \Delta\left[\frac{F_{X} \sin \tilde{\varphi}}{\left(m_{\pi}^{2}-m_{\eta}^{2}\right) F_{\eta^{\prime}}}\left(\cos \tilde{\varphi}+\frac{\sqrt{2} F_{\pi}}{F_{\eta^{\prime}}} \sin \tilde{\varphi}\right)\right. \\
& \left.+\frac{F_{X} \cos \tilde{\varphi}}{\left(m_{\pi}^{2}-m_{\eta^{\prime}}^{2}\right) F_{\eta^{\prime}}}\left(\frac{\sqrt{2} F_{\pi}}{F_{\eta^{\prime}}} \cos \tilde{\varphi}-\sin \tilde{\varphi}\right)-\frac{\sqrt{2} F_{\pi} F_{X}}{\left(m_{\pi}^{2}-m_{\eta_{X}}^{2}\right) F_{\eta^{\prime}}^{2}}\right] \text {, } \\
& \delta_{1}=\frac{B \Delta}{\sqrt{3}\left(m_{\eta}^{2}-m_{\pi}^{2}\right)}\left(\cos \tilde{\varphi}+\frac{\sqrt{2} F_{\pi}}{F_{\eta^{\prime}}} \sin \tilde{\varphi}\right) \text {, } \\
& \alpha_{1}=\cos \tilde{\varphi}, \quad \beta_{1}=\frac{F_{\pi}}{F_{\eta^{\prime}}} \sin \tilde{\varphi}, \quad \gamma_{1}=\frac{\sqrt{3} F_{X}}{F_{\eta^{\prime}}} \sin \tilde{\varphi}, \\
& \delta_{2}=\frac{B \Delta}{\sqrt{3}\left(m_{\eta^{\prime}}^{2}-m_{\pi}^{2}\right)}\left(\frac{\sqrt{2} F_{\pi}}{F_{\eta^{\prime}}} \cos \tilde{\varphi}-\sin \tilde{\varphi}\right) \text {, } \\
& \alpha_{2}=-\sin \tilde{\varphi}, \quad \beta_{2}=\frac{F_{\pi}}{F_{\eta^{\prime}}} \cos \tilde{\varphi}, \quad \gamma_{2}=\frac{\sqrt{3} F_{X}}{F_{\eta^{\prime}}} \cos \tilde{\varphi}, \\
& \delta_{3}=\frac{\sqrt{2} B \Delta F_{X}}{\left(m_{\eta_{X}}^{2}-m_{\pi}^{2}\right) F_{\eta^{\prime}}}, \\
& \alpha_{3}=0, \quad \beta_{3}=\frac{\sqrt{3} F_{X}}{F_{\eta^{\prime}}}, \quad \gamma_{3}=-\frac{F_{\pi}}{F_{\eta^{\prime}}},
\end{aligned}
$$

where $m_{\pi}^{2}, m_{\eta}^{2}, m_{\eta^{\prime}}^{2}, m_{\eta_{X}}^{2}$ are given by Eqs. (2.26), (2.29), (2.30), (2.31). The only modifications with respect to the "unperturbed" matrix $\mathbf{C}_{0}$, reported in Eq. (2.28), are in the elements $\alpha_{0}, \beta_{0}, \gamma_{0}, \delta_{1}, \delta_{2}, \delta_{3}$, which are now different from zero and of order $\Delta$ : in the limit $\Delta \rightarrow 0$ the matrix $\mathbf{C}$ correctly reduces to the matrix $\mathbf{C}_{0}$.

At the end of the previous subsection we had observed that in the case $\Delta=0$ the last 
term $\delta \mathcal{L}_{4}^{(c)}$ in the four-meson Lagrangian (3.6) , being proportional to $\eta_{X}^{4}$, contributes only to the elastic scattering amplitude $\eta_{X} \eta_{X} \rightarrow \eta_{X} \eta_{X}$. Instead, in the realistic case in which $\Delta \equiv m_{u}-m_{d} \neq 0$, this term has the form (obtained using Eqs. (3.10)-(3.11) derived above):

$$
\delta \mathcal{L}_{4}^{(c)}=\frac{c}{6}\left(\frac{\sqrt{3}}{F_{\pi}} S_{\pi}-\frac{1}{F_{X}} S_{X}\right)^{4}=\frac{c}{6}\left[\left(\frac{\sqrt{2} B \Delta}{\left(m_{\pi}^{2}-m_{\eta_{X}}^{2}\right) F_{\pi}}\right) \pi^{0}+\left(\frac{F_{\eta^{\prime}}}{F_{\pi} F_{X}}\right) \eta_{X}\right]^{4} .
$$

Therefore, when $\Delta \neq 0$ this term contributes also to the decay $\eta_{X} \rightarrow 3 \pi^{0}$, but this contribution is of order $\mathcal{O}\left(\Delta^{3}\right)$, and therefore it is strongly suppressed, for small $\Delta$, when compared with the similar contributions derived from the other terms in the Lagrangian (3.6) [see Eq. (3.21) below]. Therefore, in the following we shall neglect this contribution.

\subsection{Decays $\eta, \eta^{\prime}, \eta_{X} \rightarrow 3 \pi^{0}, \pi^{+} \pi^{-} \pi^{0}$}

In this section we shall evaluate the leading-order (LO) amplitudes and the corresponding widths for the decays of $\eta, \eta^{\prime}$ and $\eta_{X}$ into $3 \pi^{0}$ or $\pi^{+} \pi^{-} \pi^{0}$. The fields in the four-meson Lagrangian $\mathcal{L}_{4}$, written in Eq. (3.6), can be expressed in terms of the fields of the physical eigenstates (which diagonalize the squared mass matrix $\mathcal{K}$ ) by using Eqs. (3.10)-(3.11). Let us start considering the decay $\eta \rightarrow 3 \pi^{0}$. As we have already said after Eq. (3.4), the first term (containing field derivatives) of the four-meson Lagrangian $\mathcal{L}_{4}$ in Eq. (3.6) does not contribute to this decay amplitude, which, therefore, turns out to be simply a constant, i.e., not dependent on the particle momenta, and given by, at the first order in the parameter $\Delta$ :

$$
\begin{aligned}
& A(\eta\left.\rightarrow 3 \pi^{0}\right)=\left\langle\pi^{0} \pi^{0} \pi^{0}\left|\mathcal{L}_{4}\right| \eta\right\rangle \\
& \quad=\frac{B}{\sqrt{3} F_{\pi}^{2}}\left\{\Delta\left(\alpha_{1}+\sqrt{2} \beta_{1}\right)+2 \sqrt{3} \tilde{m}\left[\delta_{1}+\left(\alpha_{1}+\sqrt{2} \beta_{1}\right)\left(\alpha_{0}+\sqrt{2} \beta_{0}\right)\right]\right\} .
\end{aligned}
$$

Using the expressions (3.11) for $\alpha_{1}, \beta_{1}, \delta_{1}, \alpha_{0}, \beta_{0}$ and expanding up to the first order in the quark masses, we obtain the following expression:

$$
A\left(\eta \rightarrow 3 \pi^{0}\right)=\frac{B \Delta}{\sqrt{3} F_{\pi}^{2}}\left[\cos \tilde{\varphi}+\frac{\sqrt{2} F_{\pi}}{F_{\eta^{\prime}}} \sin \tilde{\varphi}\right]
$$


The amplitude for the decay $\eta^{\prime} \rightarrow 3 \pi^{0}$ can be obtained by simply substituting $\left(\delta_{1}, \alpha_{1}, \beta_{1}\right)$ with $\left(\delta_{2}, \alpha_{2}, \beta_{2}\right)$ in the expression (3.13). We thus obtain the following expression:

$$
A\left(\eta^{\prime} \rightarrow 3 \pi^{0}\right)=\frac{B \Delta}{\sqrt{3} F_{\pi}^{2}}\left[\frac{\sqrt{2} F_{\pi}}{F_{\eta^{\prime}}} \cos \tilde{\varphi}-\sin \tilde{\varphi}\right]
$$

Let us observe that in the limit $F_{X} \rightarrow 0$ (that is, $F_{\eta^{\prime}} \rightarrow F_{\pi}$ ) the expressions (3.14) and (3.15) correctly reduce to the corresponding expressions derived by Di Vecchia et al. in Ref. [23], i.e.:

$$
\begin{aligned}
\left.A\left(\eta \rightarrow 3 \pi^{0}\right)\right|_{F_{X}=0} & =\frac{B \Delta}{\sqrt{3} F_{\pi}^{2}}(\cos \varphi+\sqrt{2} \sin \varphi), \\
\left.A\left(\eta^{\prime} \rightarrow 3 \pi^{0}\right)\right|_{F_{X}=0} & =\frac{B \Delta}{\sqrt{3} F_{\pi}^{2}}(\sqrt{2} \cos \varphi-\sin \varphi)
\end{aligned}
$$

where $\varphi$ is the mixing angle without the contribution coming from the new $U(1)$ axial condensate, and it is given by Eq. (1.8) with $F_{X}=0$, i.e., $\tan \varphi=\frac{\sqrt{2}}{9 A} B F_{\pi}^{2}\left(m_{s}-\tilde{m}\right)=$ $\frac{F_{\pi}^{2}}{6 \sqrt{2} A}\left(m_{\eta}^{2}-m_{\pi}^{2}\right)$.

From the amplitudes (3.14) and (3.15), we can derive the corresponding decay widths by integrating over the final-state phase space, according to the formula (valid for constant amplitudes $A$ and three identical final particles) $\Gamma=\frac{1}{2 M} \int \frac{1}{3 !} \mathrm{d} \Phi^{(3)}|A|^{2}=\frac{|A|^{2}}{2 M \cdot 3 !} \Phi^{(3)}$, where the total phase space $\Phi^{(3)}$ is given by (see, for example, Ref. [30] and references therein):

$$
\Phi^{(3)}=\int \frac{\mathrm{d} s \mathrm{~d} t}{128 \pi^{3} M^{2}}=\frac{1}{128 \pi^{3} M^{2}} \int_{s_{2}}^{s_{3}} \frac{\mathrm{d} s}{s} \sqrt{\left(s-s_{1}\right)\left(s-s_{2}\right)\left(s_{3}-s\right)\left(s_{4}-s\right)}
$$

where $M$ is the mass of the initial particle and $s_{1} \equiv\left(m_{1}-m_{2}\right)^{2}, s_{2} \equiv\left(m_{1}+m_{2}\right)^{2}$, $s_{3} \equiv\left(M-m_{3}\right)^{2}, s_{4} \equiv\left(M+m_{3}\right)^{2}, m_{1}, m_{2}$ and $m_{3}$ being the masses of the three final particles; $s$ and $t$ are the usual Mandelstam variables, defined as $s \equiv\left(P-p_{1}\right)^{2}$ and $t \equiv\left(P-p_{2}\right)^{2}$, where $P$ is the four-momentum of the initial particle and $p_{1}, p_{2}, p_{3}$ are the four-momenta of the three final particles $\left(P^{2}=M^{2}, p_{1}^{2}=m_{1}^{2}, p_{2}^{2}=m_{2}^{2}, p_{3}^{2}=m_{3}^{2}\right)$.

After performing numerically the integration in Eq. (3.18) for the two cases that we are considering, using the values for the meson masses as reported by the Particle Data Group 
[24], we have obtained the following expressions for the decay widths:

$$
\begin{aligned}
\Gamma_{\mathrm{LO}}\left(\eta \rightarrow 3 \pi^{0}\right) & =\frac{(B \Delta)^{2}}{36 F_{\pi}^{4}}\left(\cos \tilde{\varphi}+\frac{\sqrt{2} F_{\pi}}{F_{\eta^{\prime}}} \sin \tilde{\varphi}\right)^{2} \frac{\Phi^{(3)}}{m_{\eta}}, \quad \frac{\Phi^{(3)}}{m_{\eta}}=9.82 \mathrm{keV},(3.1) \\
\Gamma_{\mathrm{LO}}\left(\eta^{\prime} \rightarrow 3 \pi^{0}\right) & =\frac{(B \Delta)^{2}}{36 F_{\pi}^{4}}\left(\frac{\sqrt{2} F_{\pi}}{F_{\eta^{\prime}}} \cos \tilde{\varphi}-\sin \tilde{\varphi}\right)^{2} \frac{\Phi^{(3)}}{m_{\eta^{\prime}}}, \quad \frac{\Phi^{(3)}}{m_{\eta^{\prime}}}=67.00 \mathrm{keV} .(3.2)
\end{aligned}
$$

Proceeding analogously, the following expression is obtained for the leading-order amplitude of the decay $\eta_{X} \rightarrow 3 \pi^{0}$ :

$$
A\left(\eta_{X} \rightarrow 3 \pi^{0}\right)=\frac{\sqrt{2} B \Delta F_{X}}{F_{\pi}^{2} F_{\eta^{\prime}}} .
$$

Let us observe that this amplitude correctly reduces to zero when $F_{X} \rightarrow 0$, i.e., when the new $U(1)$ axial condensate is zero. Concerning the derivation of the decay width, the mass $m_{\eta_{X}}$ of the exotic meson $\eta_{X}$ is not directly known (but see the discussion at the end of this subsection) and therefore the integration in Eq. (3.18) cannot be performed numerically. However, on the basis of what we have said in the previous section, the mass of the $\eta_{X}$ is expected to be quite large, at least larger than the mass of the $\eta^{\prime}$. So it is probably not a too bad approximation to neglect the meson masses in the total phase space for this process. In the limit $m_{1}=m_{2}=m_{3}=0$ Eq. (3.18) reduces to

$$
\Phi_{0}^{(3)}(M)=\frac{M^{2}}{256 \pi^{3}}
$$

and for the width of the decay $\eta_{X} \rightarrow 3 \pi^{0}$ we obtain the following approximate expression:

$$
\Gamma_{\mathrm{LO}}\left(\eta_{X} \rightarrow 3 \pi^{0}\right)=\left|A\left(\eta_{X} \rightarrow 3 \pi^{0}\right)\right|^{2} \frac{\Phi_{0}^{(3)}\left(m_{\eta_{X}}\right)}{2 m_{\eta_{X}} \cdot 3 !}=\frac{(B \Delta)^{2} F_{X}^{2}}{1536 \pi^{3} F_{\pi}^{4} F_{\eta^{\prime}}^{2}} m_{\eta_{X}}
$$

Let us now study the decays of $\eta, \eta^{\prime}$ and $\eta_{X}$ into $\pi^{+} \pi^{-} \pi^{0}$. As we have already observed above, also the four-meson Lagrangian term $\delta \mathcal{L}_{4}^{(f)}$, defined in Eq. (3.4) and containing derivatives of the fields, gives contributions to the amplitudes of these decays. In 
particular, one finds that:

$$
\begin{aligned}
\delta A^{(f)}\left(\eta \rightarrow \pi^{+} \pi^{-} \pi^{0}\right) & =\left\langle\pi^{+} \pi^{-} \pi^{0}\left|\delta \mathcal{L}_{4}^{(f)}\right| \eta\right\rangle=\frac{1}{F_{\pi}^{2}} \delta_{0} \delta_{1}\left(s-s_{0}\right), \\
\delta A^{(f)}\left(\eta^{\prime} \rightarrow \pi^{+} \pi^{-} \pi^{0}\right) & =\left\langle\pi^{+} \pi^{-} \pi^{0}\left|\delta \mathcal{L}_{4}^{(f)}\right| \eta^{\prime}\right\rangle=\frac{1}{F_{\pi}^{2}} \delta_{0} \delta_{2}\left(s-s_{0}^{\prime}\right), \\
\delta A^{(f)}\left(\eta_{X} \rightarrow \pi^{+} \pi^{-} \pi^{0}\right) & =\left\langle\pi^{+} \pi^{-} \pi^{0}\left|\delta \mathcal{L}_{4}^{(f)}\right| \eta_{X}\right\rangle=\frac{1}{F_{\pi}^{2}} \delta_{0} \delta_{3}\left(s-\bar{s}_{0}\right),
\end{aligned}
$$

where the coefficients $\delta_{0}, \delta_{1}, \delta_{2}$ and $\delta_{3}$ are defined in Eqs. (3.10)-(3.11), while $s_{0}, s_{0}^{\prime}$ and $\bar{s}_{0}$ are so defined:

$$
s_{0} \equiv \frac{1}{3}\left(m_{\eta}^{2}+3 m_{\pi}^{2}\right), \quad s_{0}^{\prime} \equiv \frac{1}{3}\left(m_{\eta^{\prime}}^{2}+3 m_{\pi}^{2}\right), \quad \bar{s}_{0} \equiv \frac{1}{3}\left(m_{\eta_{X}}^{2}+3 m_{\pi}^{2}\right),
$$

and, as usual, $s \equiv\left(P-P_{\pi^{0}}\right)^{2}=\left(P_{\pi^{+}}+P_{\pi^{-}}\right)^{2}, P$ being the four-momentum of the initial particle $\left(\eta, \eta^{\prime}, \eta_{X}\right)$ and $P_{\pi^{0}}, P_{\pi^{+}}, P_{\pi^{-}}$being the four-momenta of the three final pions. Adding also the contributions coming from the second term in the r.h.s. of Eq. (3.6), we obtain the following expressions for the amplitudes of the decays $\eta, \eta^{\prime}, \eta_{X} \rightarrow \pi^{+} \pi^{-} \pi^{0}$ :

$$
\begin{aligned}
A\left(\eta \rightarrow \pi^{+} \pi^{-} \pi^{0}\right) & =\frac{B \Delta}{3 \sqrt{3} F_{\pi}^{2}}\left(\cos \tilde{\varphi}+\frac{\sqrt{2} F_{\pi}}{F_{\eta^{\prime}}} \sin \tilde{\varphi}\right)\left[1+\frac{3\left(s-s_{0}\right)}{m_{\eta}^{2}-m_{\pi}^{2}}\right], \\
A\left(\eta^{\prime} \rightarrow \pi^{+} \pi^{-} \pi^{0}\right) & =\frac{B \Delta}{3 \sqrt{3} F_{\pi}^{2}}\left(\frac{\sqrt{2} F_{\pi}}{F_{\eta^{\prime}}} \cos \tilde{\varphi}-\sin \tilde{\varphi}\right)\left[1+\frac{3\left(s-s_{0}^{\prime}\right)}{m_{\eta^{\prime}}^{2}-m_{\pi}^{2}}\right], \\
A\left(\eta_{X} \rightarrow \pi^{+} \pi^{-} \pi^{0}\right) & =\frac{\sqrt{2} B \Delta F_{X}}{3 F_{\pi}^{2} F_{\eta^{\prime}}}\left[1+\frac{3\left(s-\bar{s}_{0}\right)}{m_{\eta_{X}}^{2}-m_{\pi}^{2}}\right] .
\end{aligned}
$$

From these amplitudes we can derive the corresponding decay widths by integrating over the final-state phase space, according to the formula (see, for example, Ref. [30] and references therein):

$$
\begin{aligned}
\Gamma & =\frac{1}{2 M} \int \mathrm{d} \Phi^{(3)}|A|^{2}=\frac{1}{2 M} \int \frac{\mathrm{d} s \mathrm{~d} t}{128 \pi^{3} M^{2}}|A|^{2} \\
& =\frac{1}{256 \pi^{3} M^{3}} \int_{s_{2}}^{s_{3}} \frac{\mathrm{d} s}{s}|A(s)|^{2} \sqrt{\left(s-s_{1}\right)\left(s-s_{2}\right)\left(s_{3}-s\right)\left(s_{4}-s\right)},
\end{aligned}
$$

where the notation is the same already used in Eq. (3.18). After performing numerically the integration in Eq. (3.31), using the values for the meson masses as reported by the 
Particle Data Group [24], we have obtained the following expressions for the decay widths:

$$
\begin{aligned}
\Gamma_{\mathrm{LO}}\left(\eta \rightarrow \pi^{+} \pi^{-} \pi^{0}\right) & =\frac{(B \Delta)^{2}}{54 F_{\pi}^{4}}\left(\cos \tilde{\varphi}+\frac{\sqrt{2} F_{\pi}}{F_{\eta^{\prime}}} \sin \tilde{\varphi}\right)^{2} \times 10.48 \mathrm{keV}, \\
\Gamma_{\mathrm{LO}}\left(\eta^{\prime} \rightarrow \pi^{+} \pi^{-} \pi^{0}\right) & =\frac{(B \Delta)^{2}}{54 F_{\pi}^{4}}\left(\frac{\sqrt{2} F_{\pi}}{F_{\eta^{\prime}}} \cos \tilde{\varphi}-\sin \tilde{\varphi}\right)^{2} \times 83.95 \mathrm{keV} .
\end{aligned}
$$

Concerning the case of the decay $\eta_{X} \rightarrow \pi^{+} \pi^{-} \pi^{0}$, we proceed exactly as for the case of the decay $\eta_{X} \rightarrow 3 \pi^{0}$ and we neglect the meson masses in the calculation of the integral (3.31), so obtaining the following approximate expression for the decay width:

$$
\Gamma_{\mathrm{LO}}\left(\eta_{X} \rightarrow \pi^{+} \pi^{-} \pi^{0}\right)=\frac{(B \Delta)^{2} F_{X}^{2}}{1536 \pi^{3} F_{\pi}^{4} F_{\eta^{\prime}}^{2}} m_{\eta_{X}}
$$

We now numerically compute our theoretical expressions for the leading-order decay widths, using for the mixing angle $\tilde{\varphi}$ the value derived from Eq. (1.8).

All our isospin-violating decay widths are proportional to the factor:

$$
(B \Delta)^{2}=m_{\pi}^{4}\left(\frac{m_{u}-m_{d}}{m_{u}+m_{d}}\right)^{2}=m_{\pi}^{4}\left(\frac{R-1}{R+1}\right)^{2} \simeq 2.66 \times 10^{7} \mathrm{MeV}^{4},
$$

where $m_{\pi}^{2}=B\left(m_{u}+m_{d}\right) \simeq(134.98 \mathrm{MeV})^{2}$ and $R \equiv m_{u} / m_{d} \simeq 0.558$ is the ratio between the up and down quark masses, determined using Eqs. (2.23) and the experimental values of the meson masses reported in the Particle Data Group [24].

We are, of course, particularly interested in the effects due to a non-zero value of the parameter $F_{X}$, related to the new $U(1)$ axial condensate considered in this paper. In Table 1 we report, for each decay process of the form $\eta, \eta^{\prime} \rightarrow 3 \pi$, the leading-order theoretical prediction, using for the parameter $F_{X}$ the value $F_{X}=24(7) \mathrm{MeV}$, that we have found studying the radiative decays $\eta, \eta^{\prime} \rightarrow \gamma \gamma$ [see the Introduction and, in particular, Eq. (1.9)]. These values are compared with the corresponding values obtained for $F_{X}=0$, i.e., in the absence of the new $U(1)$ axial condensate (in Table 1 we also explicitly show the correction to the decay widths, $\Delta \Gamma_{\mathrm{LO}} \equiv \Gamma_{\mathrm{LO}}\left(F_{X}=24 \pm 7 \mathrm{MeV}\right)-\Gamma_{\mathrm{LO}}\left(F_{X}=0\right)$, coming from a non-zero value of $F_{X}$ ), and also with the experimental values.

Concerning the comparison with the experimental values, it is well known that, because of large unitarity corrections due to strong final-state interactions, one has to go beyond 


\begin{tabular}{l|c|cc|c}
\hline Decay & $\Gamma_{\text {exp }}(\mathrm{keV})$ & \multicolumn{2}{|c|}{$\Gamma_{\mathrm{LO}}(\mathrm{keV})$} & $\Delta \Gamma_{\mathrm{LO}}(\mathrm{keV})$ \\
& & $F_{X}=0$ & $F_{X}=24(7) \mathrm{MeV}$ & \\
\hline \hline & & & & \\
$\eta \rightarrow 3 \pi^{0}$ & $0.423(26)$ & 0.178 & $0.176(1)$ & $-0.002(1)$ \\
$\eta^{\prime} \rightarrow 3 \pi^{0}$ & $0.33(6)$ & 0.84 & $0.62(10)$ & $-0.24(10)$ \\
$\eta \rightarrow \pi^{+} \pi^{-} \pi^{0}$ & $0.30(2)$ & 0.127 & $0.125(1)$ & $-0.002(1)$ \\
$\eta^{\prime} \rightarrow \pi^{+} \pi^{-} \pi^{0}$ & $0.70(25)$ & 0.70 & $0.52(8)$ & $-0.18(8)$ \\
& & & & \\
\hline
\end{tabular}

Table 1: The leading-order theoretical predictions for the decay widths, computed both for $F_{X}=0$ and for $F_{X}=24(7) \mathrm{MeV}$, and the corresponding corrections to the decay widths, $\Delta \Gamma_{\mathrm{LO}} \equiv \Gamma_{\mathrm{LO}}\left(F_{X}=24 \pm 7 \mathrm{MeV}\right)-\Gamma_{\mathrm{LO}}\left(F_{X}=0\right)$, compared with the experimental values.

leading and even one-loop order in chiral perturbation theory in order to obtain a valid, reliable representation of the $\eta, \eta^{\prime} \rightarrow 3 \pi$ decay amplitudes and of the corresponding decay widths, that can be successfully compared with the experimental values [31, 32, 33, 34, 35, 36.

In the present paper we are not, of course, aiming at that. In particular, we cannot proceed as in the case of the radiative decays $\eta, \eta^{\prime} \rightarrow \gamma \gamma$, i.e., we cannot extract the value of $F_{X}$ (and of the mixing angle $\tilde{\varphi}$ ) by comparing, e.g., the leading-order theoretical predictions (3.19) and (3.20), for the $\eta \rightarrow 3 \pi^{0}$ and $\eta^{\prime} \rightarrow 3 \pi^{0}$ decay widths, with the corresponding experimental values reported in Table 1. Indeed, making use of Eq. (1.8) for $\tan \tilde{\varphi}$, one easily verifies that, being $\tan \tilde{\varphi}$ and $F_{\eta^{\prime}} \equiv \sqrt{F_{\pi}^{2}+3 F_{X}^{2}}$ increasing functions of $F_{X}$, the expression (3.19) for $\Gamma_{\mathrm{LO}}\left(\eta \rightarrow 3 \pi^{0}\right)$ is a decreasing functions of $F_{X}$ : so, being its value at $F_{X}=0$ already smaller than the corresponding experimental value, it turns out that there is no value of $F_{X}$ which makes the expression (3.19) compatible with the experimental value in Table $1 * *$

Instead, our aim is simply to quantify the corrections coming from a non-zero value of the parameter $F_{X}$, taking the leading-order amplitudes/widths in the $F_{X}=0$ case as a useful reference point. From the values reported in Table 1 we can conclude that:

i) In the case of the $\eta \rightarrow 3 \pi$ decays, the size of the corrections $\Delta \Gamma_{\text {LO }}$ coming from a

${ }^{*}$ Even considering the singlet decay constant $F_{\eta^{\prime}}$ and the mixing angle $\tilde{\varphi}$ in Eqs. (3.19) and (3.20) as free parameters, to be fixed from a comparison with the experimental values reported in Table 1, we would find a too small value $F_{\eta^{\prime}} \simeq 68 \mathrm{MeV}$ for the singlet decay constant, incompatible with the formula (1.7), i.e., $F_{\eta^{\prime}}=\sqrt{F_{\pi}^{2}+3 F_{X}^{2}} \geq F_{\pi}=92.2(4) \mathrm{MeV}$, and also an anomalously large value $\tilde{\varphi} \simeq 44^{\circ}$ for the mixing angle. 
non-zero value $F_{X}=24(7) \mathrm{MeV}$, with respect to the $F_{X}=0$ case, is very small, being of the order of $1 \%$, i.e., comparable to (or even smaller than) the size of the electromagnetic corrections for these decays, which have been recently re-calculated in Ref. [37.

ii) Instead, in the case of the $\eta^{\prime} \rightarrow 3 \pi$ decays, the size of the corrections $\Delta \Gamma_{\mathrm{LO}}$ is much larger, being of the order of $30 \%$. Moreover, at least for the decay $\eta^{\prime} \rightarrow 3 \pi^{0}$ (where the statistical errors are smaller), this (negative) correction seems to go in the right direction, improving the agreement between the theoretical prediction and the experimental value.

Concerning the decays of the $\eta_{X}$ into three pions, we derive the following relations between its mass $m_{\eta_{X}}$ and the decay widths:

$$
\frac{\Gamma_{\mathrm{LO}}\left(\eta_{X} \rightarrow 3 \pi^{0}\right)}{m_{\eta_{X}}}=\frac{\Gamma_{\mathrm{LO}}\left(\eta_{X} \rightarrow \pi^{+} \pi^{-} \pi^{0}\right)}{m_{\eta_{X}}}=\left(4.35_{-1.97}^{+2.17}\right) \times 10^{-7} .
$$

These constraints could be used to identify a possible candidate for the exotic singlet meson $\eta_{X}$, once we know its mass and decay widths. According to the Particle Data Group [24], the possible candidates for the $\eta_{X}$, having the same quantum numbers $I^{G}\left(J^{P C}\right)=$ $0^{+}\left(0^{-+}\right)$of the $\eta^{\prime}$, but a larger mass, are the following:

$$
\begin{array}{lll}
\eta(1295): & \Gamma_{\text {tot }}=55(5) \mathrm{MeV}, \\
\eta(1405): & \Gamma_{\text {tot }}=51(3) \mathrm{MeV}, \\
\eta(1475): & \Gamma_{\text {tot }}=85(9) \mathrm{MeV}, \\
\eta(1760): & \Gamma_{\text {tot }}=96(70) \mathrm{MeV}, \\
\eta(2225): & \Gamma_{\text {tot }}=185_{-40}^{+70} \mathrm{MeV} .
\end{array}
$$

Unfortunately, no quantitative determination of their decay widths into three pions has been done up to now.

\subsection{Decays $\eta^{\prime} \rightarrow \eta \pi \pi$ and $\eta_{X} \rightarrow \eta \pi \pi, \eta^{\prime} \pi \pi$}

We now study the decays of $\eta^{\prime}$ into $\eta \pi^{0} \pi^{0}, \eta \pi^{+} \pi^{-}$and of $\eta_{X}$ into $\eta \pi^{0} \pi^{0}, \eta \pi^{+} \pi^{-}, \eta^{\prime} \pi^{0} \pi^{0}$, $\eta^{\prime} \pi^{+} \pi^{-}$. This decays do not violate isospin and so they can happen also when $\Delta=0$. 
Therefore, in order to evaluate the amplitudes and the corresponding widths for these decays, we shall use the approximate expressions (2.28) of the eigenstates at the order zero in the isospin-violating parameter $\Delta$.

The following expression is obtained for the leading-order amplitudes of the decays $\eta^{\prime} \rightarrow$ $\eta \pi^{0} \pi^{0}$ and $\eta^{\prime} \rightarrow \eta \pi^{+} \pi^{-}$(which, in the limit of exact $S U(2)_{V}$ isospin symmetry, are equal):

$$
\begin{aligned}
A\left(\eta^{\prime}\right. & \left.\rightarrow \eta \pi^{0} \pi^{0}\right)=A\left(\eta^{\prime} \rightarrow \eta \pi^{+} \pi^{-}\right) \\
& =\frac{m_{\pi}^{2}}{6 F_{\pi}^{2}}\left[\frac{2 \sqrt{2} F_{\pi}}{F_{\eta^{\prime}}} \cos (2 \tilde{\varphi})+\left(\frac{2 F_{\pi}^{2}}{F_{\eta^{\prime}}^{2}}-1\right) \sin (2 \tilde{\varphi})\right] .
\end{aligned}
$$

In the limit $F_{X} \rightarrow 0$ these amplitudes reduce to the expression already found in Ref. [23], i.e.:

$$
\left.A\left(\eta^{\prime} \rightarrow \eta \pi^{0} \pi^{0}\right)\right|_{F_{X}=0}=\left.A\left(\eta^{\prime} \rightarrow \eta \pi^{+} \pi^{-}\right)\right|_{F_{X}=0}=\frac{m_{\pi}^{2}}{6 F_{\pi}^{2}}[2 \sqrt{2} \cos (2 \varphi)+\sin (2 \varphi)] .
$$

After numerical integration of the phace space (3.18), using the values for the meson masses reported in Ref. [24], we obtain the corresponding decay widths:

$$
\begin{aligned}
\Gamma_{\mathrm{LO}}\left(\eta^{\prime} \rightarrow \eta \pi^{0} \pi^{0}\right) & =\left|A\left(\eta^{\prime} \rightarrow \eta \pi^{0} \pi^{0}\right)\right|^{2} \frac{\Phi^{(3)}}{2 m_{\eta^{\prime}} \cdot 2 !}, \quad \frac{\Phi^{(3)}}{2 m_{\eta^{\prime}} \cdot 2 !}=1.093 \mathrm{keV}, \\
\Gamma_{\mathrm{LO}}\left(\eta^{\prime} \rightarrow \eta \pi^{+} \pi^{-}\right) & =\left|A\left(\eta^{\prime} \rightarrow \eta \pi^{+} \pi^{-}\right)\right|^{2} \frac{\Phi^{(3)}}{2 m_{\eta^{\prime}}}=2 \Gamma_{\mathrm{LO}}\left(\eta^{\prime} \rightarrow \eta \pi^{0} \pi^{0}\right) .
\end{aligned}
$$

We proceed as in the previous subsection and numerically compute our theoretical expressions for the leading-order decay widths, using for the mixing angle $\tilde{\varphi}$ the value derived from Eq. (1.8) and for the parameter $F_{X}$ the value $F_{X}=24(7) \mathrm{MeV}$, that we have found studying the radiative decays $\eta, \eta^{\prime} \rightarrow \gamma \gamma$. Again, our aim is simply to quantify the corrections coming from a non-zero value of the parameter $F_{X}$, taking the leading-order amplitudes/widths in the $F_{X}=0$ case as a reference point. In this case, however, it is already known from Ref. [23] that the leading-order theoretical predictions for $F_{X}=0$,

$$
\left.\Gamma_{\mathrm{LO}}\left(\eta^{\prime} \rightarrow \eta \pi^{+} \pi^{-}\right)\right|_{F_{X}=0}=\left.2 \Gamma_{\mathrm{LO}}\left(\eta^{\prime} \rightarrow \eta \pi^{0} \pi^{0}\right)\right|_{F_{X}=0}=2.42 \mathrm{keV},
$$

are in strong disagreement with the experimental values [24], $\Gamma_{\exp }\left(\eta^{\prime} \rightarrow \eta \pi^{+} \pi^{-}\right)=84(5)$ $\mathrm{keV}$ and $\Gamma_{\exp }\left(\eta^{\prime} \rightarrow \eta \pi^{0} \pi^{0}\right)=42(4) \mathrm{keV}$.

We can try to see if the introduction of a non-zero value of $F_{X}$ can cure, at least in part, 
the strong disagreement between leading-order theoretical predictions and experimental values: however, the answer to this question is negative. In fact, we find that:

$$
\left.\Gamma_{\mathrm{LO}}\left(\eta^{\prime} \rightarrow \eta \pi^{+} \pi^{-}\right)\right|_{F_{X}=24(7) \mathrm{MeV}}=\left.2 \Gamma_{\mathrm{LO}}\left(\eta^{\prime} \rightarrow \eta \pi^{0} \pi^{0}\right)\right|_{F_{X}=24(7) \mathrm{MeV}}=1.78(30) \mathrm{keV}
$$

Even if the correction $\Delta \Gamma_{\mathrm{LO}}$ is quite large (of the order of $30 \%$ ) if compared with the value of $\Gamma_{\mathrm{LO}}$ at $F_{X}=0$, it is, however, too small if compared with the experimental value. In addition, the correction $\Delta \Gamma_{\mathrm{LO}}$, being negative, goes in the "wrong" direction, lowering the theoretical prediction at $F_{X}=0$, which is already much smaller than the experimental value: in other words, it is not possible to find a value of the parameter $F_{X}$ which moves the leading-order theoretical prediction towards the experimental value. Moreover, the amplitude (3.38) is a constant, while the experimental data are well fitted by a nonconstant amplitude having the form: $A\left(\eta^{\prime} \rightarrow \eta \pi \pi\right)=A\left(1-\sigma_{1} T_{\eta}\right)$, where $T_{\eta}$ is the kinetic energy of the $\eta, A$ and $\sigma_{1}$ are some constants. As already observed in Ref. [23], in order to describe this behaviour, and to obtain a better agreement with the experimental value of the decay width, it is not enough to retain only the leading order in the $1 / N$ expansion, but one has to go to the next-to-leading order, adding to the Lagrangian (1.2) non-leading terms such as $\lambda Q^{2} \operatorname{Tr}\left(\partial_{\mu} U \partial^{\mu} U^{\dagger}\right)$, that may be very important because of the proportionality of the leading terms to the tiny pion mass $\dagger$ The systematic introduction, in our model, of higher-order terms in the $1 / N$ expansion (including also one-loop graphs, which are of order $1 / N^{2}$ : see, for example, Refs. [31, 39]) is, of course, a quite hard task, which is beyond the aim of the present paper (but it will probably be addressed in a subsequent work).

Concerning the exotic meson $\eta_{X}$, the following expressions are obtained for the leadingorder amplitudes of the decays $\eta_{X} \rightarrow \eta \pi \pi$ and $\eta_{X} \rightarrow \eta^{\prime} \pi \pi$ :

$$
\begin{aligned}
& A\left(\eta_{X} \rightarrow \eta \pi^{0} \pi^{0}\right)=A\left(\eta_{X} \rightarrow \eta \pi^{+} \pi^{-}\right)=\frac{\sqrt{2} m_{\pi}^{2} F_{X}}{\sqrt{3} F_{\pi}^{2} F_{\eta^{\prime}}}\left(\cos \tilde{\varphi}+\frac{\sqrt{2} F_{\pi}}{F_{\eta^{\prime}}} \sin \tilde{\varphi}\right) \\
& A\left(\eta_{X} \rightarrow \eta^{\prime} \pi^{0} \pi^{0}\right)=A\left(\eta_{X} \rightarrow \eta^{\prime} \pi^{+} \pi^{-}\right)=\frac{\sqrt{2} m_{\pi}^{2} F_{X}}{\sqrt{3} F_{\pi}^{2} F_{\eta^{\prime}}}\left(\frac{\sqrt{2} F_{\pi}}{F_{\eta^{\prime}}} \cos \tilde{\varphi}-\sin \tilde{\varphi}\right) .
\end{aligned}
$$

From these amplitudes we can obtain the corresponding decay widths, using, for the integrated phase space (3.18), the following approximate expression obtained neglecting

\footnotetext{
${ }^{\dagger}$ A different and alternative approach, first suggested in Ref. [38, considers the decay $\eta^{\prime} \rightarrow \eta \pi \pi$ to be dominated by coupling to nearby scalar resonances.
} 
the pion masses (while retaining the $\eta$ and $\eta^{\prime}$ masses different from zero):

$$
\Phi_{1}^{(3)}(M, m)=\frac{M^{4}-m^{4}+4 M^{2} m^{2} \ln (m / M)}{256 \pi^{3} M^{2}},
$$

where $M$ is the mass of the initial particle and $m$ is the mass of the final massive particle. We thus find the following expressions:

$$
\begin{aligned}
& \Gamma_{\mathrm{LO}}\left(\eta_{X} \rightarrow \eta \pi^{0} \pi^{0}\right)=\left|A\left(\eta_{X} \rightarrow \eta \pi^{0} \pi^{0}\right)\right|^{2} \frac{\Phi_{1}^{(3)}\left(m_{\eta_{X}}, m_{\eta}\right)}{2 m_{\eta_{X}} \cdot 2 !} \\
& =\frac{m_{\pi}^{4} F_{X}^{2}}{1536 \pi^{3} F_{\pi}^{4} F_{\eta^{\prime}}^{2}}\left(\cos \tilde{\varphi}+\frac{\sqrt{2} F_{\pi}}{F_{\eta^{\prime}}} \sin \tilde{\varphi}\right)^{2}\left[m_{\eta_{X}}-\frac{m_{\eta}^{4}}{m_{\eta_{X}}^{3}}+\frac{4 m_{\eta}^{2}}{m_{\eta_{X}}} \ln \left(\frac{m_{\eta}}{m_{\eta_{X}}}\right)\right] \\
& \quad=\left(0.95_{-0.42}^{+0.46}\right) \times 10^{-5} m_{\eta_{X}}\left[1-\left(\frac{m_{\eta}}{m_{\eta_{X}}}\right)^{4}+4\left(\frac{m_{\eta}}{m_{\eta_{X}}}\right)^{2} \ln \left(\frac{m_{\eta}}{m_{\eta_{X}}}\right)\right], \\
& \Gamma_{\mathrm{LO}}\left(\eta_{X} \rightarrow \eta \pi^{+} \pi^{-}\right)=\left|A\left(\eta_{X} \rightarrow \eta \pi^{+} \pi^{-}\right)\right|^{2} \frac{\Phi_{1}^{(3)}\left(m_{\eta_{X}}, m_{\eta}\right)}{2 m_{\eta_{X}}} \\
& \quad=2 \Gamma_{\mathrm{LO}}\left(\eta_{X} \rightarrow \eta \pi^{0} \pi^{0}\right),
\end{aligned}
$$

and also:

$$
\begin{aligned}
& \Gamma_{\mathrm{LO}}\left(\eta_{X} \rightarrow \eta^{\prime} \pi^{0} \pi^{0}\right)=\left|A\left(\eta_{X} \rightarrow \eta^{\prime} \pi^{0} \pi^{0}\right)\right|^{2} \frac{\Phi_{1}^{(3)}\left(m_{\eta_{X}}, m_{\eta^{\prime}}\right)}{2 m_{\eta_{X}} \cdot 2 !} \\
& =\frac{m_{\pi}^{4} F_{X}^{2}}{1536 \pi^{3} F_{\pi}^{4} F_{\eta^{\prime}}^{2}}\left(\frac{\sqrt{2} F_{\pi}}{F_{\eta^{\prime}}} \cos \tilde{\varphi}-\sin \tilde{\varphi}\right)^{2}\left[m_{\eta_{X}}-\frac{m_{\eta^{\prime}}^{4}}{m_{\eta_{X}}^{3}}+\frac{4 m_{\eta^{\prime}}^{2}}{m_{\eta_{X}}} \ln \left(\frac{m_{\eta^{\prime}}}{m_{\eta_{X}}}\right)\right] \\
& \quad=\left(0.49_{-0.18}^{+0.12}\right) \times 10^{-5} m_{\eta_{X}}\left[1-\left(\frac{m_{\eta^{\prime}}}{m_{\eta_{X}}}\right)^{4}+4\left(\frac{m_{\eta^{\prime}}}{m_{\eta_{X}}}\right)^{2} \ln \left(\frac{m_{\eta^{\prime}}}{m_{\eta_{X}}}\right)\right], \\
& \Gamma_{\mathrm{LO}}\left(\eta_{X} \rightarrow \eta^{\prime} \pi^{+} \pi^{-}\right)=\left|A\left(\eta_{X} \rightarrow \eta^{\prime} \pi^{+} \pi^{-}\right)\right|^{2} \frac{\Phi_{1}^{(3)}\left(m_{\eta_{X}}, m_{\eta^{\prime}}\right)}{2 m_{\eta_{X}}} \\
& \quad=2 \Gamma_{\mathrm{LO}}\left(\eta_{X} \rightarrow \eta^{\prime} \pi^{0} \pi^{0}\right) .
\end{aligned}
$$

As in the case of Eq. (3.36), also these relations could in principle be used to identify a possible candidate for the exotic singlet meson $\eta_{X}$, once we know its mass and decay widths. However, a certain caution must be used since, as in the case of the decays $\eta^{\prime} \rightarrow \eta \pi \pi$, large corrections to these leading-order results could come from non-leading terms in the $1 / N$ expansion: only a detailed analysis of our model at the next-to-leading order in $1 / N$ shall clarify this point. 
3.5. Possible decays $\eta_{X} \rightarrow 3 \eta, \eta \eta \eta^{\prime}, \eta \eta^{\prime} \eta^{\prime}, 3 \eta^{\prime}$ ?

If the exotic singlet meson $\eta_{X}$ were heavy enough, let us say, if $m_{\eta_{X}}>3 m_{\eta} \simeq 1640$ $\mathrm{MeV}$, it could also decay into three $\eta$ particles. The amplitude for this decay, which does not violate $S U(2)_{V}$ isospin, can be evaluated at the order zero in the isospin-violating parameter $\Delta$, so using the approximate form (2.28) for the physical eigenstates, and the following result is obtained:

$$
A\left(\eta_{X} \rightarrow 3 \eta\right)=\frac{8 \sqrt{2} m_{K}^{2} F_{X}}{3 \sqrt{3} F_{\pi}^{2} F_{\eta^{\prime}}}\left(-\cos \tilde{\varphi}+\frac{3 \sqrt{2} F_{\pi}}{2 F_{\eta^{\prime}}} \sin \tilde{\varphi}\right) .
$$

In order to estimate the decay width, considering that also the final-state particles $\eta$ are rather heavy, we use the approximate expression for the total phase space (3.18) in the non-relativistic limit, i.e.:

$$
\Phi_{n r}^{(3)}\left(M, m_{1}, m_{2}, m_{3}\right)=\frac{Q^{2}}{64 \pi^{2}} \sqrt{\frac{m_{1} m_{2} m_{3}}{\left(m_{1}+m_{2}+m_{3}\right)^{3}}},
$$

where $Q \equiv M-m_{1}-m_{2}-m_{3}$ is the so-called " $Q$ value" of the decay.

We thus obtain the following approximate expression for the decay width:

$$
\begin{aligned}
\Gamma_{\mathrm{LO}} & \left(\eta_{X} \rightarrow 3 \eta\right)=\left|A\left(\eta_{X} \rightarrow 3 \eta\right)\right|^{2} \frac{\Phi_{n r}^{(3)}\left(m_{\eta_{X}}, m_{\eta}, m_{\eta}, m_{\eta}\right)}{2 m_{\eta_{X}} \cdot 3 !} \\
& =\frac{m_{K}^{4} F_{X}^{2}}{486 \sqrt{3} \pi^{2} F_{\pi}^{4} F_{\eta^{\prime}}^{2}}\left(\cos \tilde{\varphi}-\frac{3 \sqrt{2} F_{\pi}}{2 F_{\eta^{\prime}}} \sin \tilde{\varphi}\right)^{2} \frac{\left(m_{\eta_{X}}-3 m_{\eta}\right)^{2}}{m_{\eta_{X}}} \\
& =\left(0.96_{-0.43}^{+0.46}\right) \times 10^{-3} m_{\eta_{X}}\left(1-\frac{3 m_{\eta}}{m_{\eta_{X}}}\right)^{2},
\end{aligned}
$$

where, as usual, we have used for the parameter $F_{X}$ the value $F_{X}=24(7) \mathrm{MeV}$, that we have found studying the radiative decays $\eta, \eta^{\prime} \rightarrow \gamma \gamma$, and for the mixing angle $\tilde{\varphi}$ the value derived from Eq. (1.8). (For example, for a value $m_{\eta_{X}} \approx 2 \mathrm{GeV}$, one would get $\Gamma_{\mathrm{LO}}\left(\eta_{X} \rightarrow 3 \eta\right) \approx 61 \mathrm{keV}$.)

Other possible decays of this kind (supposing that the $\eta_{X}$ is heavy enough so that they are kinematically allowed) are $\eta_{X} \rightarrow \eta \eta \eta^{\prime}, \eta \eta^{\prime} \eta^{\prime}, 3 \eta^{\prime}$, and their amplitudes and corresponding widths can be derived in a similar way. 


\section{Conclusions}

In this paper we have considered a scenario (supported by some lattice results) in which a $U(1)$-breaking condensate survives across the chiral transition at $T_{c h}$, staying different from zero up to $T_{U(1)}>T_{c h}$. This scenario has important consequences for the pseudoscalar-meson sector, which can be studied using an effective Lagrangian model, including also the new $U(1)$ chiral condensate. This model, originally proposed in Refs. [6, 17, 8, 9] and elaborated in Refs. [10, 11, 12], could perhaps be verified in the near future by heavy-ion experiments, by analysing the pseudoscalar-meson spectrum in the singlet sector.

Section 2 contains a brief review (for the benefit of the reader) of the main results, obtained in the original papers [6, 8, 10], concerning the mass spectrum of the Chiral Effective Lagrangian. The Lagrangian (2.1) contains a new field $X$ and three new parameters, namely $F_{X}, \omega_{1}$ and $c_{1}$, with respect to the usual Lagrangian of Witten, Di Vecchia, Veneziano et al. In this paper we have assumed that the parameter $F_{X}$, which is essentially proportional to the new $U(1)$ axial condensate, is different from zero. In this case, there are two singlet pseudoscalar mesons, the $\eta^{\prime}$ and an exotic particle $\eta_{X}$, whose squared masses (assuming also that the coupling constant $c_{1}$ of the interaction term $\operatorname{det}(U) X^{\dagger}+\operatorname{det}\left(U^{\dagger}\right) X$ in Eq. (1.3) , between the usual $q \bar{q}$ meson field $U$ and the exotic meson field $X$, is different from zero and not too small: see the discussion in Appendix B) are given by Eqs. (2.30) and (2.31): in particular, the exotic particle $\eta_{X}$ turns out to have a large (non-chiral) mass term of order $\mathcal{O}(1)$ in the large $-N$ limit, generated by the (non-zero) coupling constant $c_{1}$.

In section 3, generalizing the results obtained in Refs. [11, 12], where the effects of the new $U(1)$ chiral condensate on the radiative decays of the pseudoscalar mesons $\eta$ and $\eta^{\prime}$ into two photons had been investigated, we have studied the effects of the $U(1)$ chiral condensate on the strong decays of the "light" pseudoscalar mesons, i.e., $\eta, \eta^{\prime} \rightarrow 3 \pi^{0}$; $\eta, \eta^{\prime} \rightarrow \pi^{+} \pi^{-} \pi^{0} ; \eta^{\prime} \rightarrow \eta \pi^{0} \pi^{0} ; \eta^{\prime} \rightarrow \eta \pi^{+} \pi^{-} ;$and also on the strong decays of the exotic ("heavy") $S U(3)$-singlet pseudoscalar state $\eta_{X}: \eta_{X} \rightarrow 3 \pi^{0} ; \eta_{X} \rightarrow \pi^{+} \pi^{-} \pi^{0} ; \eta_{X} \rightarrow \eta \pi^{0} \pi^{0}$; $\eta_{X} \rightarrow \eta \pi^{+} \pi^{-} ; \eta_{X} \rightarrow \eta^{\prime} \pi^{0} \pi^{0} ; \eta_{X} \rightarrow \eta^{\prime} \pi^{+} \pi^{-} ; \eta_{X} \rightarrow 3 \eta, 3 \eta^{\prime}, \eta \eta \eta^{\prime}, \eta \eta^{\prime} \eta^{\prime}$. Concerning the decays of the exotic particle $\eta_{X}$, we have found some relations between its mass and its decay widths, which in principle might be useful to identify a possible candidate for this

particle. According to the Particle Data Group [24], the possible candidates for the $\eta_{X}$, having the same quantum numbers $I^{G}\left(J^{P C}\right)=0^{+}\left(0^{-+}\right)$of the $\eta^{\prime}$, but a larger mass, are, 
at the moment, those reported in Eq. (3.37) (other candidates with larger masses are also present, but some of their quantum numbers $I^{G}\left(J^{P C}\right)$ are not yet known): unfortunately, no quantitative determination of their decay widths into (e.g.) three pions has been done up to now.

Concerning the decays $\eta, \eta^{\prime} \rightarrow 3 \pi$, it is well known that, because of large unitarity corrections due to strong final-state interactions, one has to go beyond leading and even one-loop order in chiral perturbation theory in order to obtain a valid, reliable representation of the decay amplitudes and of the corresponding decay widths, that can be successfully compared with the experimental values [31, 32, 33, 34, 35, 36].

In the present paper we have not, of course, aimed at that. In particular, we could not proceed as in the case of the radiative decays $\eta, \eta^{\prime} \rightarrow \gamma \gamma$, i.e., we could not extract the value of $F_{X}$ (and of the mixing angle $\tilde{\varphi}$ ) by comparing, e.g., the leading-order theoretical predictions (3.19) and (3.20), for the $\eta \rightarrow 3 \pi^{0}$ and $\eta^{\prime} \rightarrow 3 \pi^{0}$ decay widths, with the corresponding experimental values reported in Table 1 of section 3 .

Instead, our aim has been simply to quantify the corrections coming from the non-zero value $F_{X}=24(7) \mathrm{MeV}$, that we have found studying the radiative decays $\eta, \eta^{\prime} \rightarrow \gamma \gamma$ [see the Introduction and in particular Eq. (1.9)], taking the leading-order amplitudes/widths in the $F_{X}=0$ case as a useful reference point. From the values reported in Table 1 of section 3 we have concluded that:

i) In the case of the $\eta \rightarrow 3 \pi$ decays, the size of the corrections $\Delta \Gamma_{\text {LO }}$ coming from a non-zero value $F_{X}=24(7) \mathrm{MeV}$, with respect to the $F_{X}=0$ case, is very small, being of the order of $1 \%$, i.e., comparable to (or even smaller than) the size of the electromagnetic corrections for these decays, which have been recently re-calculated in Ref. [37.

ii) Instead, in the case of the $\eta^{\prime} \rightarrow 3 \pi$ decays, the size of the corrections $\Delta \Gamma_{\mathrm{LO}}$ is much larger, being of the order of $30 \%$. Moreover, at least for the decay $\eta^{\prime} \rightarrow 3 \pi^{0}$ (where the statistical errors are smaller), this (negative) correction seems to go in the right direction, improving the agreement between the theoretical prediction and the experimental value.

Finally, concerning the decays $\eta^{\prime} \rightarrow \eta \pi^{0} \pi^{0}$ and $\eta^{\prime} \rightarrow \eta \pi^{+} \pi^{-}$, knowing already from Ref. [23] that the leading-order theoretical predictions for $F_{X}=0$ are in strong disagreement with the experimental values, we have tried to see if the introduction of a non-zero value 
of $F_{X}$ can cure, at least in part, this disagreement: but we have found that it cannot. In fact, even if the correction $\Delta \Gamma_{\mathrm{LO}}$ is quite large (of the order of $30 \%$ ) if compared with the value of $\Gamma_{\mathrm{LO}}$ at $F_{X}=0$, it is, however, too small if compared with the experimental value, and, moreover, being negative, it goes in the "wrong" direction, lowering the theoretical prediction at $F_{X}=0$, which is already much smaller than the experimental value. (In other words, it is not possible to find a value of the parameter $F_{X}$ which moves the leading-order theoretical prediction towards the experimental value.)

However, as we have already stressed in the conclusions of Refs. [11, 12], one should keep in mind that our results have been derived from a very simplified model, obtained by doing a first-order expansion in $1 / N$ and in the quark masses. We expect that such a model can furnish only qualitative or, at most, "semi-quantitative" predictions. As already observed in Ref. [23], in order to obtain a better agreement with the experimental data of the decay widths, most probably it is not enough to retain only the leading order in the $1 / N$ expansion, but one has to go to the next-to-leading order. The introduction, in our model, of higher-order terms in the $1 / N$ expansion is, of course, a quite hard task, which is beyond the aim of the present paper. Further studies are therefore necessary in order to continue this analysis from a more quantitative point of view. We expect that some progress will be made along this line in the near future.

\section{Acknowledgements}

The author is extremely grateful to D. Somensi for his help during the initial stage of the work. 


\section{Appendix A: The $U(1)$ chiral order parameter}

We make the assumption (discussed in the Introduction) that the $U(1)$ chiral symmetry is broken independently from the $S U(L) \otimes S U(L)$ symmetry. The usual chiral order parameter $\langle\bar{q} q\rangle$ is an order parameter both for $S U(L) \otimes S U(L)$ and for $U(1)_{A}$ : when it is different from zero, $S U(L) \otimes S U(L)$ is broken down to $S U(L)_{V}$ ("V" stands for "vectorial") and also $U(1)_{A}$ is broken. In fact, under a $U(1)$ chiral transformation with parameter $\alpha$ (as usual, $q_{L} \equiv \frac{1}{2}\left(1+\gamma_{5}\right) q$ and $q_{R} \equiv \frac{1}{2}\left(1-\gamma_{5}\right) q$, with $\gamma_{5} \equiv-i \gamma^{0} \gamma^{1} \gamma^{2} \gamma^{3}$, denote respectively the left-handed and the right-handed quark fields):

$$
U(1)_{A}: \quad q \rightarrow e^{-i \alpha \gamma_{5}} q, \quad \text { i.e., } \quad q_{L} \rightarrow e^{-i \alpha} q_{L}, \quad q_{R} \rightarrow e^{i \alpha} q_{R},
$$

the chiral condensate would transform as (assuming the $U(1)_{A}$ symmetry to be realized à la Wigner-Weyl):

$$
U(1)_{A}: \quad\langle\bar{q} q\rangle \rightarrow e^{2 i \alpha}\left\langle\bar{q}_{L} q_{R}\right\rangle+e^{-2 i \alpha}\left\langle\bar{q}_{R} q_{L}\right\rangle
$$

By taking $\alpha=\pi / 2$, we would obtain $\langle\bar{q} q\rangle \rightarrow-\langle\bar{q} q\rangle$ : therefore, if the chiral condensate is different from zero, the $U(1)_{A}$ symmetry cannot be realized $\grave{a}$ la Wigner-Weyl. Thus we need another quantity which could be an order parameter only for the $U(1)$ chiral symmetry [6, 7, 8, 9]. The most simple quantity of this kind was introduced by Kobayashi and Maskawa in 1970 [16], as an additional effective vertex in a generalized Nambu-JonaLasinio model, and it was later derived by 'tHooft in 1976 [3], as an instanton-induced quark interaction. (See also Ref. [17] for an historical review on this subject.)

For a theory with $L$ light quark flavours (of mass $m_{i} \ll \Lambda_{Q C D} ; i=1, \ldots, L$ ), it is a $2 L$-fermion interaction that has the chiral transformation properties of:

$$
\mathcal{O}_{U(1)}^{(L)} \sim \operatorname{det}\left[\bar{q}_{s t}\left(\frac{1+\gamma_{5}}{2}\right) q_{t}\right]+\text { H.c. }=\operatorname{det}_{s t}\left(\bar{q}_{s R} q_{t L}\right)+\operatorname{det}_{s t}\left(\bar{q}_{s L} q_{t R}\right)
$$

where $s, t=1, \ldots, L$ are flavour indices, but the colour indices are arranged in a more general way (see below). Since under chiral $U(L) \otimes U(L)$ transformations the quark fields transform as follows:

$$
U(L) \otimes U(L): \quad q_{L} \rightarrow V_{L} q_{L} \quad, \quad q_{R} \rightarrow V_{R} q_{R}
$$


where $V_{L}$ and $V_{R}$ are arbitrary $L \times L$ unitary matrices, we immediately derive the transformation property of $\mathcal{O}_{U(1)}^{(L)}$ under $U(L) \otimes U(L)$ :

$$
U(L) \otimes U(L): \quad \mathcal{O}_{U(1)}^{(L)} \rightarrow \operatorname{det}\left(V_{L}\right) \operatorname{det}\left(V_{R}\right)^{*} \operatorname{det}_{s t}\left(\bar{q}_{s R} q_{t L}\right)+\text { H.c. }
$$

This just means that $\mathcal{O}_{U(1)}^{(L)}$ is invariant under $S U(L) \otimes S U(L) \otimes U(1)_{V}$, while it is not invariant under the $U(1)_{A}$ transformation (A.1):

$$
U(1)_{A}: \quad \mathcal{O}_{U(1)}^{(L)} \rightarrow e^{-i 2 L \alpha} \operatorname{det}_{s t}\left(\bar{q}_{s R} q_{t L}\right)+\text { H.c. }
$$

\section{A.1 The $U(1)$ chiral condensate for $L=2$}

As an example let us consider the most simple case, that is $L=2$, but with a general colour group $S U(N)$. It is not hard to find (using the Fierz relations both for the spinorial matrices and the $S U(N)$ generators in their fundamental representation) that the most general colour-singlet, Hermitian and $P$-invariant local quantity (without derivatives) which has the required chiral transformation properties is just the following four-fermion local operator:

$$
\mathcal{O}_{U(1)}^{(L=2)}\left(\alpha_{0}, \beta_{0}\right)=F_{b d}^{a c}\left(\alpha_{0}, \beta_{0}\right) \epsilon^{s t}\left(\bar{q}_{1 R}^{a} q_{s L}^{b} \cdot \bar{q}_{2 R}^{c} q_{t L}^{d}+\bar{q}_{1 L}^{a} q_{s R}^{b} \cdot \bar{q}_{2 L}^{c} q_{t R}^{d}\right)
$$

where the colour tensor $F_{b d}^{a c}\left(\alpha_{0}, \beta_{0}\right)$ is given by:

$$
F_{b d}^{a c}\left(\alpha_{0}, \beta_{0}\right)=\alpha_{0} \delta_{b}^{a} \delta_{d}^{c}+\beta_{0} \delta_{d}^{a} \delta_{b}^{c}
$$

$\alpha_{0}$ and $\beta_{0}$ being arbitrary real parameters. In Eq. (A.7), $a, b, c, d \in\{1, \ldots, N\}$ are colour indices; $s, t \in\{1,2\}$ are flavour indices and $\epsilon^{s t}=-\epsilon^{t s}, \epsilon^{12}=1$. Dirac indices are contracted between the first and the second fermion field and also between the third and the fourth one. Note that if we choose $\alpha_{0}=N$ and $\beta_{0}=-1, \mathcal{O}_{U(1)}^{(L=2)}\left(\alpha_{0}, \beta_{0}\right)$ just becomes (up to a proportionality constant) the effective Lagrangian for two flavours of quarks in an instanton background, found by 'tHooft in [3] .

Now, to obtain an order parameter for the $U(1)$ chiral symmetry, one can simply take the vacuum expectation value of $\mathcal{O}_{U 1)}^{(L=2)}\left(\alpha_{0}, \beta_{0}\right)$ :

$$
C_{U(1)}^{(L=2)}\left(\alpha_{0}, \beta_{0}\right) \equiv\left\langle\mathcal{O}_{U(1)}^{(L=2)}\left(\alpha_{0}, \beta_{0}\right)\right\rangle
$$


The arbitrarity in the choice of $\alpha_{0}$ and $\beta_{0}$ (indeed of only one of them, since only their ratio is relevant) can be removed if we require that the new $U(1)$ chiral condensate is "independent", in a sense which will be explained below, of the usual chiral condensate $\langle\bar{q} q\rangle$. As it was pointed out by Shifman, Vainshtein and Zakharov in [40], a matrix element of the form $\left\langle\bar{q} \Gamma_{1} q \cdot \bar{q} \Gamma_{2} q\right\rangle$ has, in general, a contribution proportional to the square of the vacuum expectation value (v.e.v.) of $\bar{q}$. This contribution corresponds to retaining the vacuum intermediate state in all the channels and neglecting the contributions of all the other states; we call this contribution the "disconnected part" of the original matrix element:

$$
\left\langle\bar{q} \Gamma_{1} q \cdot \bar{q} \Gamma_{2} q\right\rangle_{d i s c}=\frac{1}{G^{2}}\left[\left(\operatorname{Tr} \Gamma_{1} \cdot \operatorname{Tr} \Gamma_{2}\right)-\operatorname{Tr}\left(\Gamma_{1} \Gamma_{2}\right)\right]\langle\bar{q} q\rangle^{2}
$$

where the normalization factor $G$ is defined as $\left(\bar{q} q=\sum_{A} \bar{q}_{A} q_{A}\right)$ :

$$
\left\langle\bar{q}_{A} q_{B}\right\rangle=\frac{\delta_{A B}}{G}\langle\bar{q} q\rangle, \quad \text { i.e., } \quad G=\delta_{A A},
$$

and the subscripts $A, B$ are collective indices which include spin, colour and flavour; therefore, $G=4 \times L \times N$ for a general $L$, and $G=8 N$ for $L=2$. When considering the operator $\mathcal{O}_{U(1)}^{(L=2)}\left(\alpha_{0}, \beta_{0}\right)$ defined in Eqs. (A.7) and (A.8), we find the following expression for its disconnected part:

$$
\left\langle\mathcal{O}_{U(1)}^{(L=2)}\left(\alpha_{0}, \beta_{0}\right)\right\rangle_{d i s c}=\frac{1}{16 N}\left[N\left(2 \alpha_{0}+\beta_{0}\right)+\left(\alpha_{0}+2 \beta_{0}\right)\right]\langle\bar{q} q\rangle^{2},
$$

where: $\langle\bar{q} q\rangle=\langle\bar{u} u\rangle+\langle\bar{d} d\rangle$. From this last equation we immediately see that the disconnected part of the condensate $C_{U(1)}^{(L=2)}\left(\alpha_{0}, \beta_{0}\right)$ vanishes with the following particular choice of the coefficients $\alpha_{0}$ and $\beta_{0}$ (only their ratio is really relevant):

$$
\frac{\beta_{0}}{\alpha_{0}}=-\frac{2 N+1}{N+2}
$$

In other words, the condensate (A.9) with $\alpha_{0}$ and $\beta_{0}$ satisfying the constraint (A.13) does not take contributions from the usual chiral condensate $\langle\bar{q} q\rangle$. To summarize, a good choice for a $U(1)$ chiral condensate which is really "independent" of the usual chiral condensate $\langle\bar{q} q\rangle$ is the following one (apart from an irrelevant multiplicative constant):

$$
C_{U(1)}^{(L=2)}=\left\langle\left(\delta_{b}^{a} \delta_{d}^{c}-\frac{2 N+1}{N+2} \delta_{d}^{a} \delta_{b}^{c}\right) \epsilon^{s t}\left(\bar{q}_{1 R}^{a} q_{s L}^{b} \cdot \bar{q}_{2 R}^{c} q_{t L}^{d}+\bar{q}_{1 L}^{a} q_{s R}^{b} \cdot \bar{q}_{2 L}^{c} q_{t R}^{d}\right)\right\rangle
$$


As a remark, we observe that the condensate $C_{U(1)}^{(L=2)}$ so defined turns out to be of order $\mathcal{O}\left(g^{2} N^{2}\right)=\mathcal{O}(N)$ in the large $-N$ expansion (this result was also derived in Ref. [7] by simply requiring that the $1 / N$ expansion of the relevant QCD Ward Identities remains well defined when including this new condensate). In the case of physical interest, i.e., $N=3$, the condensate Eq. (A.14) becomes:

$$
C_{U(1)}^{(L=2)}=\left\langle\left(\delta_{b}^{a} \delta_{d}^{c}-\frac{7}{5} \delta_{d}^{a} \delta_{b}^{c}\right) \epsilon^{s t}\left(\bar{q}_{1 R}^{a} q_{s L}^{b} \cdot \bar{q}_{2 R}^{c} q_{t L}^{d}+\bar{q}_{1 L}^{a} q_{s R}^{b} \cdot \bar{q}_{2 L}^{c} q_{t R}^{d}\right)\right\rangle
$$

\section{A.2 The $U(1)$ chiral condensate for $L=3$}

So far we have considered the most simple case $L=2$. However, this procedure can be easily generalized to every $L$, and we can take as an order parameter for the $U(1)$ chiral symmetry:

$$
C_{U(1)}^{(L)}=\left\langle\mathcal{O}_{U(1)}^{(L)}\right\rangle
$$

As we have done in the case $L=2$, the colour indices may be arranged in such a way that the $U(1)$ chiral condensate does not take contributions from the usual chiral condensate $\langle\bar{q} q\rangle$ : as a consenquence of this, the new condensate will be of order $\mathcal{O}\left(g^{2 L-2} N^{L}\right)=\mathcal{O}(N)$ in the large $-N$ expansion [7, 8].

In the real-world case there are $L=3$ light flavours, $u, d$, and $s$, with masses $m_{u}, m_{d}$, and $m_{s}$ which are small compared to the QCD mass-scale $\Lambda_{Q C D}$. Proceeding as in the case $L=2$ [see Eq. (A.7)], one reduces to consider the following general colour-singlet, Hermitian and $P$-invariant local six-fermion operator (without derivatives):

$$
\mathcal{O}_{U(1)}^{(L=3)}=F_{b_{1} b_{2} b_{3}}^{a_{1} a_{2} a_{3}} \epsilon^{l_{1} l_{2} l_{3}} \bar{q}_{1 R}^{a_{1}} q_{l_{1} L}^{b_{1}} \cdot \bar{q}_{2 R}^{a_{2}} q_{l_{2} L}^{b_{2}} \cdot \bar{q}_{3 R}^{a_{3}} q_{l_{3} L}^{b_{3}}+\text { H.c. },
$$

where $a_{1}, a_{2}, a_{3}, b_{1}, b_{2}, b_{3} \in\{1,2, \cdots N\}$ are colour indices, $l_{1}, l_{2}, l_{3} \in\{1,2,3\}$ are flavour indices and the colour tensor $F_{b_{1} b_{2} b_{3}}^{a_{1} a_{2} a_{3}}$ is given by:

$$
\begin{aligned}
F_{b_{1} b_{2} b_{3}}^{a_{1} a_{2} a_{3}} & =\alpha_{1} \delta_{b_{1}}^{a_{1}} \delta_{b_{2}}^{a_{2}} \delta_{b_{3}}^{a_{3}}+\alpha_{2} \delta_{b_{2}}^{a_{1}} \delta_{b_{3}}^{a_{2}} \delta_{b_{1}}^{a_{3}}+\alpha_{3} \delta_{b_{3}}^{a_{1}} \delta_{b_{1}}^{a_{2}} \delta_{b_{2}}^{a_{3}} \\
& +\beta_{1} \delta_{b_{2}}^{a_{1}} \delta_{b_{1}}^{a_{2}} \delta_{b_{3}}^{a_{3}}+\beta_{2} \delta_{b_{1}}^{a_{1}} \delta_{b_{3}}^{a_{2}} \delta_{b_{2}}^{a_{3}}+\beta_{3} \delta_{b_{3}}^{a_{1}} \delta_{b_{2}}^{a_{2}} \delta_{b_{1}}^{a_{3}},
\end{aligned}
$$

with $\alpha_{1}, \alpha_{2}, \alpha_{3}, \beta_{1}, \beta_{2}, \beta_{3}$ real parameters. However, differently from the case $L=2$, the operator $\mathcal{O}_{U(1)}^{(L=3)}$ in Eqs. (A.17) - (A.18), with arbitrary real parameters $\alpha_{1}, \alpha_{2}, \alpha_{3}, \beta_{1}, \beta_{2}, \beta_{3}$, is not, in general, invariant under a $S U(3) \otimes S U(3)$ chiral transformation:

$$
S U(3) \otimes S U(3): \quad q_{L} \rightarrow U_{L} q_{L} \quad, \quad q_{R} \rightarrow U_{R} q_{R}
$$


( $\operatorname{det} U_{L}=\operatorname{det} U_{R}=1$ ). Invariance under $S U(3) \otimes S U(3)$ is, instead, recovered provided that the colour tensor $F_{b_{1} b_{2} b_{3}}^{a_{1} a_{2} a_{3}}$ satisfies the following symmetry property:

$$
F_{b_{1} b_{2} b_{3}}^{a_{1} a_{2} a_{3}}=F_{b_{i} b_{j} b_{k}}^{a_{i} a_{j} a_{k}}, \quad \forall \text { permutations }\{i, j, k\} \text { of }\{1,2,3\}
$$

In fact, in this case it is easy to see that the operator (A.17) can be re-written in the following form:

$$
\mathcal{O}_{U(1)}^{(L=3)}=F_{b_{1} b_{2} b_{3}}^{a_{1} a_{2} a_{3}} \frac{1}{3 !} \epsilon^{r_{1} r_{2} r_{3}} \epsilon^{l_{1} l_{2} l_{3}} \bar{q}_{r_{1} R}^{a_{1}} q_{l_{1} L}^{b_{1}} \cdot \bar{q}_{r_{2} R}^{a_{2}} q_{l_{2} L}^{b_{2}} \cdot \bar{q}_{r_{3} R}^{a_{3}} q_{l_{3} L}^{b_{3}}+\text { H.c. }
$$

which is manifestly invariant under $S U(3) \otimes S U(3)$ :

$$
\begin{aligned}
& \mathcal{O}_{U(1)}^{(L=3)} \rightarrow F_{b_{1} b_{2} b_{3}}^{a_{1} a_{2} a_{3}} \frac{1}{3 !} \epsilon^{r_{1} r_{2} r_{3}} \epsilon^{l_{1} l_{2} l_{3}} \bar{q}_{s_{1} R}^{a_{1}}\left(U_{R}^{\dagger}\right)_{s_{1} r_{1}}\left(U_{L}\right)_{l_{1} m_{1}} q_{m_{1} L}^{b_{1}} \\
& \text { - } \quad \bar{q}_{s_{2} R}^{a_{2}}\left(U_{R}^{\dagger}\right)_{s_{2} r_{2}}\left(U_{L}\right)_{l_{2} m_{2}} q_{m_{2} L}^{b_{2}} \cdot \bar{q}_{s_{3} R}^{a_{3}}\left(U^{\dagger}\right)_{s_{3} r_{3}}\left(U_{L}\right)_{l_{3} m_{3}} q_{m_{3} L}^{b_{3}}+\text { H.c. } \\
& =F_{b_{1} b_{2} b_{3}}^{a_{1} a_{2} a_{3}} \frac{1}{3 !} \operatorname{det}\left(U_{R}^{\dagger}\right) \epsilon^{s_{1} s_{2} s_{3}} \operatorname{det}\left(U_{L}\right) \epsilon^{m_{1} m_{2} m_{3}} \\
& \times \quad \bar{q}_{s_{1} R}^{a_{1}} q_{m_{1} L}^{b_{1}} \cdot \bar{q}_{s_{2} R}^{a_{2}} q_{m_{2} L}^{b_{2}} \cdot \bar{q}_{s_{3} R}^{a_{3}} q_{m_{3} L}^{b_{3}}+\text { H.c. } \\
& =F_{b_{1} b_{2} b_{3}}^{a_{1} a_{2} a_{3}} \frac{1}{3 !} \epsilon^{s_{1} s_{2} s_{3}} \epsilon^{m_{1} m_{2} m_{3}} \bar{q}_{s_{1} R}^{a_{1}} q_{m_{1} L}^{b_{1}} \cdot \bar{q}_{s_{2} R}^{a_{2}} q_{m_{2} L}^{b_{2}} \cdot \bar{q}_{s_{3} R}^{a_{3}} q_{m_{3} L}^{b_{3}}+\text { H.c. } \\
& =\mathcal{O}_{U(1)}^{(L=3)} \text {. }
\end{aligned}
$$

(Or, equivalently, one can start from the expression (A.21) of the six-fermion local operator, which, on the basis of (A.22), is invariant under $S U(3) \otimes S U(3)$ for every choice of the colour tensor $F_{b_{1} b_{2} b_{3}}^{a_{1} a_{2} a_{3}}$ : but one immediately recognizes that only the symmetric part of the colour tensor, satisfying the relation (A.20), contributes to the r.h.s. of (A.21), the anti-symmetric parts being trivially cancelled out. Note that, in the case $L=2$, the most general colour tensor (A.8) automatically satifies the symmetry property, $F_{b d}^{a c}=F_{d b}^{c a}$.)

The symmetry property (A.20) imposes the following constraints on the parameters of the colour tensor (A.18):

$$
\alpha_{3}=\alpha_{2}, \quad \beta_{3}=\beta_{2}=\beta_{1} .
$$

The colour tensor has, therefore, the following form:

$$
\begin{aligned}
F_{b_{1} b_{2} b_{3}}^{a_{1} a_{2} a_{3}} & =\alpha_{1} \delta_{b_{1}}^{a_{1}} \delta_{b_{2}}^{a_{2}} \delta_{b_{3}}^{a_{3}}+\alpha_{2}\left(\delta_{b_{2}}^{a_{1}} \delta_{b_{3}}^{a_{2}} \delta_{b_{1}}^{a_{3}}+\delta_{b_{3}}^{a_{1}} \delta_{b_{1}}^{a_{2}} \delta_{b_{2}}^{a_{3}}\right) \\
& +\beta_{1}\left(\delta_{b_{2}}^{a_{1}} \delta_{b_{1}}^{a_{2}} \delta_{b_{3}}^{a_{3}}+\delta_{b_{1}}^{a_{1}} \delta_{b_{3}}^{a_{2}} \delta_{b_{2}}^{a_{3}}+\delta_{b_{3}}^{a_{1}} \delta_{b_{2}}^{a_{2}} \delta_{b_{1}}^{a_{3}}\right)
\end{aligned}
$$


in terms of three arbitrary real parameters $\alpha_{1}, \alpha_{2}, \beta_{1}$.

Let us now evaluate the vacuum expectation value of the operator $\mathcal{O}_{U(1)}^{(L=3)}$ :

$$
\begin{aligned}
C_{U(1)}^{(L=3)} & \equiv\left\langle\mathcal{O}_{U(1)}^{(L=3)}\right\rangle=F_{b_{1} b_{2} b_{3}}^{a_{1} a_{2} a_{3}} \epsilon^{l_{1} l_{2} l_{3}}\left\langle\bar{q} \Gamma_{1} q \cdot \bar{q} \Gamma_{2} q \cdot \bar{q} \Gamma_{3} q\right\rangle+\text { c.c. } \\
& =F_{b_{1} b_{2} b_{3}}^{a_{1} a_{3}} \epsilon^{l_{1} l_{2} l_{3}}\left(\Gamma_{1}\right)_{A B}\left(\Gamma_{2}\right)_{C D}\left(\Gamma_{3}\right)_{E F}\left\langle\bar{q}_{A} q_{B} \bar{q}_{C} q_{D} \bar{q}_{E} q_{F}\right\rangle+\text { c.c. },
\end{aligned}
$$

where [see Eq. (A.17)]:

$$
\begin{aligned}
& \left(\Gamma_{1}\right)_{A B}=\left(\Gamma_{1}\right)_{i_{1} j_{1}, m_{1} n_{1}}^{c_{1} d_{1}}=\left(\frac{1+\gamma_{5}}{2}\right)_{i_{1} j_{1}} \otimes\left(\delta_{m_{1} 1} \delta_{n_{1} l_{1}}\right) \otimes\left(\delta^{c_{1} a_{1}} \delta^{d_{1} b_{1}}\right), \\
& \left(\Gamma_{2}\right)_{C D}=\left(\Gamma_{2}\right)_{i_{2} j_{2}, m_{2} n_{2}}^{c_{2} d_{2}}=\left(\frac{1+\gamma_{5}}{2}\right)_{i_{2} j_{2}} \otimes\left(\delta_{m_{2} 2} \delta_{n_{2} l_{2}}\right) \otimes\left(\delta^{c_{2} a_{2}} \delta^{d_{2} b_{2}}\right), \\
& \left(\Gamma_{3}\right)_{E F}=\left(\Gamma_{3}\right)_{i_{3} j_{3}, m_{3} n_{3}}^{c_{3} d_{3}}=\left(\frac{1+\gamma_{5}}{2}\right)_{i_{3} j_{3}} \otimes\left(\delta_{m_{3} 3} \delta_{n_{3} l_{3}}\right) \otimes\left(\delta^{c_{3} a_{3}} \delta^{d_{3} b_{3}}\right),
\end{aligned}
$$

where $i, j$ are Dirac indices, $m, n$ are flavour indices, and $c, d$ are colour indices.

As in the case $L=2$ treated above, we can write the vacuum expectation value of the operator $\mathcal{O}_{U(1)}^{(L=3)}$ as the sum of a connected part, which does not depend on the chiral condensate $\langle\bar{q} q\rangle$, and a disconnected part, which instead contains the chiral condensate $\langle\bar{q} q\rangle$, i.e., $C_{U(1)}^{(L=3)}=\left\langle\mathcal{O}_{U(1)}^{(L=3)}\right\rangle_{\text {conn }}+\left\langle\mathcal{O}_{U(1)}^{(L=3)}\right\rangle_{\text {disc }}$, where:

$$
\left\langle\mathcal{O}_{U(1)}^{(L=3)}\right\rangle_{d i s c}=F_{b_{1} b_{2} b_{3}}^{a_{1} a_{2} a_{3}} \epsilon^{l_{1} l_{2} l_{3}}\left(\Gamma_{1}\right)_{A B}\left(\Gamma_{2}\right)_{C D}\left(\Gamma_{3}\right)_{E F}\left\langle\bar{q}_{A} q_{B} \bar{q}_{C} q_{D} \bar{q}_{E} q_{F}\right\rangle_{d i s c}+\text { c.c. },
$$

and the disconnected part of the v.e.v. of the six-fermion operator has the following form:

$$
\begin{aligned}
\left\langle\bar{q}_{A} q_{B} \bar{q}_{C} q_{D} \bar{q}_{E} q_{F}\right\rangle_{d i s c} & \\
& =\left\langle\bar{q}_{A} q_{B}\right\rangle\left\langle\bar{q}_{C} q_{D} \bar{q}_{E} q_{F}\right\rangle_{c o n n}+\left\langle\bar{q}_{C} q_{D}\right\rangle\left\langle\bar{q}_{A} q_{B} \bar{q}_{E} q_{F}\right\rangle_{c o n n}+\left\langle\bar{q}_{E} q_{F}\right\rangle\left\langle\bar{q}_{A} q_{B} \bar{q}_{C} q_{D}\right\rangle_{c o n n} \\
& -\left\langle\bar{q}_{A} q_{D}\right\rangle\left\langle\bar{q}_{C} q_{B} \bar{q}_{E} q_{F}\right\rangle_{c o n n}-\left\langle\bar{q}_{A} q_{F}\right\rangle\left\langle\bar{q}_{C} q_{D} \bar{q}_{E} q_{B}\right\rangle_{c o n n}-\left\langle\bar{q}_{C} q_{B}\right\rangle\left\langle\bar{q}_{A} q_{D} \bar{q}_{E} q_{F}\right\rangle_{c o n n} \\
& -\left\langle\bar{q}_{C} q_{F}\right\rangle\left\langle\bar{q}_{A} q_{B} \bar{q}_{E} q_{D}\right\rangle_{c o n n}-\left\langle\bar{q}_{E} q_{B}\right\rangle\left\langle\bar{q}_{A} q_{F} \bar{q}_{C} q_{D}\right\rangle_{c o n n}-\left\langle\bar{q}_{E} q_{D}\right\rangle\left\langle\bar{q}_{A} q_{B} \bar{q}_{C} q_{F}\right\rangle_{c o n n} \\
& +\left\langle\bar{q}_{A} q_{B}\right\rangle\left\langle\bar{q}_{C} q_{D}\right\rangle\left\langle\bar{q}_{E} q_{F}\right\rangle-\left\langle\bar{q}_{A} q_{B}\right\rangle\left\langle\bar{q}_{C} q_{F}\right\rangle\left\langle\bar{q}_{E} q_{D}\right\rangle-\left\langle\bar{q}_{A} q_{D}\right\rangle\left\langle\bar{q}_{C} q_{B}\right\rangle\left\langle\bar{q}_{E} q_{F}\right\rangle \\
& +\left\langle\bar{q}_{A} q_{D}\right\rangle\left\langle\bar{q}_{C} q_{F}\right\rangle\left\langle\bar{q}_{E} q_{B}\right\rangle+\left\langle\bar{q}_{A} q_{F}\right\rangle\left\langle\bar{q}_{C} q_{B}\right\rangle\left\langle\bar{q}_{E} q_{D}\right\rangle-\left\langle\bar{q}_{A} q_{F}\right\rangle\left\langle\bar{q}_{C} q_{D}\right\rangle\left\langle\bar{q}_{E} q_{B}\right\rangle .
\end{aligned}
$$

On the basis of Eq. (A.11), we see that the disconnected part of the condensate (A.25) can be written as:

$$
\left\langle\mathcal{O}_{U(1)}^{(L=3)}\right\rangle_{d i s c}=A_{1}\langle\bar{q} q\rangle+A_{3}\langle\bar{q} q\rangle^{3}
$$


where the first term $\left(A_{1}\langle\bar{q} q\rangle\right)$, proportional to the chiral condensate, is originated by the first nine terms in the r.h.s. of Eq. ( by the last six terms in the r.h.s. of Eq. (A.28) and represents the completely disconnected part, proportional to the third power of the chiral condensate.

Explicitly, using Eq. (A.11), with $G=4 \times 3 \times N=12 N$, the form (A.26) of the $\Gamma$ matrices and the form (A.24) of the colour tensor $F_{b_{1} b_{2} b_{3}}^{a_{1} a_{2} a_{3}}$, satisfying the symmetry property (A.20), we obtain the following expression for the coefficient $A_{1}$ :

$$
\begin{aligned}
A_{1} & =\frac{1}{G^{3}} F_{b_{1} b_{2} b_{3}}^{a_{1} a_{2} a_{3}} \epsilon^{l_{1} l_{2} l_{3}}\left\{\operatorname{Tr} \Gamma_{1}\left\langle\bar{q} \Gamma_{2} q \cdot \bar{q} \Gamma_{3} q\right\rangle_{c o n n}\right. \\
& +\operatorname{Tr} \Gamma_{2}\left\langle\bar{q} \Gamma_{1} q \cdot \bar{q} \Gamma_{3} q\right\rangle_{c o n n}+\operatorname{Tr} \Gamma_{3}\left\langle\bar{q} \Gamma_{1} q \cdot \bar{q} \Gamma_{2} q\right\rangle_{c o n n} \\
& -\left\langle\bar{q} \Gamma_{1} \Gamma_{2} q \cdot \bar{q} \Gamma_{3} q\right\rangle_{c o n n}-\left\langle\bar{q} \Gamma_{2} \Gamma_{1} q \cdot \bar{q} \Gamma_{3} q\right\rangle_{c o n n} \\
& -\left\langle\bar{q} \Gamma_{1} \Gamma_{3} q \cdot \bar{q} \Gamma_{2} q\right\rangle_{c o n n}-\left\langle\bar{q} \Gamma_{3} \Gamma_{1} q \cdot \bar{q} \Gamma_{2} q\right\rangle_{c o n n} \\
& \left.-\left\langle\bar{q} \Gamma_{2} \Gamma_{3} q \cdot \bar{q} \Gamma_{1} q\right\rangle_{c o n n}-\left\langle\bar{q} \Gamma_{3} \Gamma_{2} q \cdot \bar{q} \Gamma_{1} q\right\rangle_{c o n n}\right\}+ \text { c.c. } \\
& =\frac{1}{12 N}\left[2 F_{b d e}^{a c e}+F_{b e d}^{a c e}+F_{\text {edb }}^{a c e}\right]\left(C_{12}^{a b c d}+C_{13}^{a b c d}+C_{23}^{a b c d}\right),
\end{aligned}
$$

where:

$$
\begin{aligned}
C_{12}^{a b c d} & \equiv \epsilon^{s t 3}\left[\left\langle\bar{q}_{1 R}^{a} q_{s L}^{b} \cdot \bar{q}_{2 R}^{c} q_{t L}^{d}\right\rangle_{c o n n}+\text { c.c. }\right], \\
C_{13}^{a b c d} & \equiv \epsilon^{s 2 t}\left[\left\langle\bar{q}_{1 R}^{a} q_{s L}^{b} \cdot \bar{q}_{3 R}^{c} q_{t L}^{d}\right\rangle_{c o n n}+\text { c.c. }\right], \\
C_{23}^{a b c d} & \equiv \epsilon^{1 s t}\left[\left\langle\bar{q}_{2 R}^{a} q_{s L}^{b} \cdot \bar{q}_{3 R}^{c} q_{t L}^{d}\right\rangle_{c o n n}+\text { c.c. }\right],
\end{aligned}
$$

and the following expression for the coefficient $A_{3}$ :

$$
\begin{aligned}
A_{3} & =\frac{2}{G^{3}} F_{b_{1} b_{2} b_{3}}^{a_{1} a_{2} a_{3}} \epsilon^{l_{1} l_{2} l_{3}}\left[\operatorname{Tr} \Gamma_{1} \operatorname{Tr} \Gamma_{2} \operatorname{Tr} \Gamma_{3}-\operatorname{Tr} \Gamma_{1} \operatorname{Tr}\left(\Gamma_{2} \Gamma_{3}\right)-\operatorname{Tr}\left(\Gamma_{1} \Gamma_{2}\right) \operatorname{Tr} \Gamma_{3}\right. \\
& \left.+\operatorname{Tr}\left(\Gamma_{1} \Gamma_{3} \Gamma_{2}\right)+\operatorname{Tr}\left(\Gamma_{1} \Gamma_{2} \Gamma_{3}\right)-\operatorname{Tr}\left(\Gamma_{1} \Gamma_{3}\right) \operatorname{Tr} \Gamma_{2}\right] \\
& =\frac{1}{216 N^{3}}\left[\alpha_{1}\left(2 N^{3}+3 N^{2}+N\right)+\alpha_{2}\left(N^{3}+6 N^{2}+5 N\right)\right. \\
& \left.+\beta_{1}\left(3 N^{3}+9 N^{2}+6 N\right)\right] .
\end{aligned}
$$

Now, if we want to obtain a new order parameter which is really independent on the usual chiral condensate $\langle\bar{q} q\rangle$, we must require that its disconnected part (A.29) vanishes independently on the value of $\langle\bar{q} q\rangle$, imposing the two conditions $A_{1}=0$ and $A_{3}=0$. Therefore, we have two independent constraints on the three parameters $\alpha_{1}, \alpha_{2}$ and $\beta_{1}$, which enter the colour tensor (A.24): the new condensate $C_{U(1)}^{(L=3)}$ is then univocally determined, apart from a multiplicative constant. 
Let us also observe that in the large $-N$ limit, taking the coefficients $\alpha_{1}, \alpha_{2}$ and $\beta_{1}$ in the colour tensor (A.24) to be of order $\mathcal{O}\left(N^{0}\right)$, the coefficient $A_{1}$ is of order $\mathcal{O}(N)$, while the coefficient $A_{3}$ is of order $\mathcal{O}\left(N^{0}\right)$ : and, consequently, the first term $A_{1}\langle\bar{q} q\rangle$ in the r.h.s. of (A.29) is of order $\mathcal{O}\left(N^{2}\right)$, while the second term $A_{3}\langle\bar{q} q\rangle^{3}$ is of order $\mathcal{O}\left(N^{3}\right)$ (being $\langle\bar{q} q\rangle=\mathcal{O}(N))$. If both these disconnected parts are zero, then the new condensate $C_{U(1)}^{(L=3)}$ is simply equal to the connected part $\left\langle\mathcal{O}_{U(1)}^{(L=3)}\right\rangle_{\text {conn }}$, which is of order $\mathcal{O}(N)$, i.e., of the same order of the usual chiral condensate $\langle\bar{q} q\rangle$ (as already observed in Refs. [7, 8]).

We also observe that the condition $A_{1}=0$ implies that the new six-fermion condensate $C_{U(1)}^{(L=3)}$ does not take contributions from four-fermion condensates of the form (A.31). In this paper we have only studied the effects of the new six-fermion $U(1)$ chiral order parameter. However, recently, four-fermion operators (which could be associated with the above-mentioned four-fermion condensates) have been used in the literature, in the study of scalar mesons, which are modelled as four-quark (i.e., $\bar{q} q \bar{q} q)$ states, called "tetraquarks" or "diquark-antidiquark" bound states [41, 42, 43]. 


\section{Appendix B: On the new parameters $F_{X}, \omega_{1}$ and $c_{1}$}

The Lagrangian (2.1) contains a new field $X$ and three new parameters, namely $F_{X}, \omega_{1}$ and $c_{1}$, with respect to the usual Lagrangian of Witten, Di Vecchia, Veneziano et al. It is therefore natural to ask if the model can be further simplified by simply eliminating some parameter. As we have already said, in this paper we are assuming that the parameter $F_{X}$, which is essentially proportional to the new $U(1)$ axial condensate, is different from zero: in section 3 we discuss the relevance of this parameter $F_{X}$ in the phenomenological analysis of the strong decays of pseudoscalar mesons.

Concerning the parameter $\omega_{1}$, we cannot say too much. We remind that the usual Lagrangian of Witten, Di Vecchia, Veneziano et al. is obtained by choosing $\omega_{1}=1$ (together with $F_{X}=0$, i.e., $X=0$ ). At low temperatures one expects that the deviations from this Lagrangian are small, in some sense, and therefore one expects that $\omega_{1}$ is not much different from 1 at low temperatures. On the other side, as already observed in Ref. [6], $\omega_{1}$ must necessarily be zero when $T \geq T_{c h}$, in order to avoid a singular behaviour of the anomalous term above the chiral transition: this implies a non trivial behaviour of $\omega_{1}$ with the temperature. However, in this paper no particular choice for the value of $\omega_{1}(T=0)$ will be done: it will be considered as a free parameter (apart from the above-mentioned limitation for $T \geq T_{c h}$ ).

The case of the parameter $c_{1}$ is much more interesting. By putting $c_{1}=0$, i.e., $c=0$ [see Eq. (2.8)], into Eqs. (2.12), these reduce to:

$$
Z_{L}=\frac{2 A\left[F_{\pi}^{2}\left(1-\omega_{1}\right)^{2}+L F_{X}^{2} \omega_{1}^{2}\right]}{F_{\pi}^{2} F_{X}^{2}}, \quad Q_{L}=0,
$$

which, when inserted into Eq. (2.11), lead to the following values for the squared masses of the two singlets $S_{1}$ and $S_{2}$ in the chiral limit:

$$
m_{S_{1}}^{2}=0, \quad m_{S_{2}}^{2}=\left(\frac{2 L A}{F_{\pi}^{2}}\right) \omega_{1}^{2}+\left(\frac{2 A}{F_{X}^{2}}\right)\left(1-\omega_{1}\right)^{2}
$$

The corresponding eigenvectors, written in terms of $S_{\pi}$ and $S_{X}$, are:

$$
\begin{aligned}
& S_{1}=\frac{1}{\sqrt{F_{\pi}^{2}\left(1-\omega_{1}\right)^{2}+L F_{X}^{2} \omega_{1}^{2}}}\left(F_{\pi}\left(\omega_{1}-1\right) S_{\pi}+\sqrt{L} F_{X} \omega_{1} S_{X}\right), \\
& S_{2}=\frac{1}{\sqrt{F_{\pi}^{2}\left(1-\omega_{1}\right)^{2}+L F_{X}^{2} \omega_{1}^{2}}}\left(\sqrt{L} F_{X} \omega_{1} S_{\pi}+F_{\pi}\left(1-\omega_{1}\right) S_{X}\right) .
\end{aligned}
$$


Let us observe that Eqs. (B.3) and (B.2) cannot be derived by simply putting $c=0$ into Eqs. (2.14) and (2.15), derived in subsection 2.1. This is due to the fact that Eqs. (2.14) and (2.15) were derived not only assuming that $c_{1} \neq 0$, but also taking the large $-N$ limit, in which the quantity $c \equiv \frac{c_{1}}{\sqrt{2}}\left(\frac{F_{X}}{\sqrt{2}}\right)\left(\frac{F_{\pi}}{\sqrt{2}}\right)^{L}$ is large, being of order $\mathcal{O}(N)$ [see Eq. (2.13)]. In that case, therefore, one obtains $Z_{L}=\mathcal{O}(1)$ and $Q_{L}=\mathcal{O}(1 / N)$, so that, from Eq. (2.11), $m_{S_{1}}^{2} \simeq \frac{Q_{L}}{Z_{L}} \simeq \frac{2 L A}{F_{\pi}^{2}+L F_{X}^{2}}=\mathcal{O}(1 / N)$ and $S_{1}$ can be identified with the particle $\eta^{\prime}$.

Instead, in the particular case in which $c_{1}=0$ [i.e., $c=0$ ], one has that $Z_{L}=\mathcal{O}(1 / N)$ and $Q_{L}=0$, so that, from Eq. (2.11), $S_{1}$ is massless (in the chiral limit) and therefore it does not verify the Witten-Veneziano formula required for the $\eta^{\prime}$. It is easy to convince oneself that, in this particular case $c_{1}=0, S_{2}$, having a squared mass of order $\mathcal{O}(1 / N)$ in the large $-N$ limit, is just the field which must be identified with the particle $\eta^{\prime}$, as required by the Witten-Veneziano mechanism for the solution of the $U(1)$ problem. In fact, by virtue of Eqs. (B.3), we can re-write the $U(1)$ axial current $J_{5, \mu}^{(L)}$, given by Eq. (2.16), in terms of the fields $S_{1}$ and $S_{2}$ :

$$
J_{5, \mu}^{(L)}=-\sqrt{2 L} \partial_{\mu}\left(F_{S_{1}} S_{1}+F_{S_{2}} S_{2}\right)
$$

where:

$$
\begin{aligned}
& F_{S_{1}}=\frac{F_{\pi}^{2}\left(\omega_{1}-1\right)+L F_{X}^{2} \omega_{1}}{\sqrt{F_{\pi}^{2}\left(1-\omega_{1}\right)^{2}+L F_{X}^{2} \omega_{1}^{2}}}, \\
& F_{S_{2}}=\frac{\sqrt{L} F_{\pi} F_{X}}{\sqrt{F_{\pi}^{2}\left(1-\omega_{1}\right)^{2}+L F_{X}^{2} \omega_{1}^{2}}},
\end{aligned}
$$

are nothing but the decay constants of the singlet pseudoscalar mesons $S_{1}$ and $S_{2}$, defined as:

$$
\begin{aligned}
& \left\langle 0\left|J_{5, \mu}^{(L)}(0)\right| S_{1}\left(\vec{p}_{1}\right)\right\rangle=i \sqrt{2 L} F_{S_{1}} p_{1 \mu}, \\
& \left\langle 0\left|J_{5, \mu}^{(L)}(0)\right| S_{2}\left(\vec{p}_{2}\right)\right\rangle=i \sqrt{2 L} F_{S_{2}} p_{2 \mu} .
\end{aligned}
$$

From Eqs. (B.2) and (B.5) one immediately verifies that the field $S_{2}$ satifies the WittenVeneziano formula, i.e.,

$$
m_{S_{2}}^{2}=\frac{2 L A}{F_{S_{2}}^{2}},
$$

and, therefore, it is nothing but the field associated with the particle $\eta^{\prime}$, with a squared (non-chiral) mass generated by the anomaly and of order $\mathcal{O}(1 / N)$ in the large- $N$ limit, 
as required by the Witten-Veneziano mechanism. (Instead, concerning the state $S_{1}$, even if, according to Eqs. (B.6) and (B.5), it is coupled to the $U(1)$ axial current, it is not coupled to the topological charge density, i.e., $\left\langle 0|Q(0)| S_{1}\left(\vec{p}_{1}\right)\right\rangle=\frac{1}{\sqrt{2 L}} F_{S_{1}} m_{S_{1}^{2}}^{2}=0$, since it is massless: therefore it does not appear as an intermediate mesonic state in the spectral decomposition of the full topological susceptibility ...)

It is interesting to observe that, in this case (differently from the case discussed in subsection 2.1), the parameter $\omega_{1}$ plays a fundamental role. In fact, when $c_{1}=0$, the anomalous Lagrangian term containing $\omega_{1}$ is the only one which generates a coupling between $U$ and $X$ (i.e., between the usual quark-antiquark pseudoscalar mesons and the exotic singlet state). By changing $\omega_{1}$ one can "move" the anomaly from $U$ to $X$. In particular, in the case $\omega_{1}=1$ the anomalous term only depends on $U$ and the field $X$ is decoupled. In this case the Lagrangian simply reduces to the sum of the usual Lagrangian written by Witten, Di Vecchia, Veneziano et al. for the field $U$ (including the anomalous term) plus a non-anomalous Lagrangian for the field $X$ : in this limit the state $S_{2}$, i.e, the $\eta^{\prime}$, reduces to the usual quark-antiquark singlet state $S_{\pi}$, while the massless state $S_{1}$ reduces to the exotic state $S_{X}$. On the contrary, in the opposite case $\omega_{1}=0$ the anomalous term only depends on the exotic field $X$ and so the state $S_{2}$, i.e, the $\eta^{\prime}$, reduces to the exotic state $S_{X}$, while the massless state $S_{1}$ reduces to the usual quark-antiquark singlet state $S_{\pi}$.

In conclusion, we have found that, in the case in which $c_{1}=0$, in addition to the usual $L^{2}-1$ non-singlet (pseudo-) Goldstone bosons and to the massive singlet $S_{2}=\eta^{\prime}$, there is another singlet $S_{1}$, which is massless in the chiral limit. This particle is therefore another (pseudo-)Goldstone boson which, when including the quark masses, should have a mass comparable with that of the other $L^{2}-1$ non-singlet pseudoscalar mesons.

In the realistic case $L=3$, by diagonalizing the squared mass matrix (2.25) with $c=0$, we derive the following expressions for the squared masses of $\eta, S_{1}$ and $S_{2}$, at the first order in the quark masses:

$$
\begin{aligned}
m_{\eta}^{2} & =\frac{1}{3} B\left\{\left(\tilde{m}+2 m_{s}\right)+\left(2 \tilde{m}+m_{s}\right) \alpha_{X}^{2}\right. \\
& +\sqrt{\left.\left[\left(\tilde{m}+2 m_{s}\right)+\left(2 \tilde{m}+m_{s}\right) \alpha_{X}^{2}\right]^{2}-36 \alpha_{X}^{2} \tilde{m} m_{s}\right\}} \\
m_{S_{1}}^{2} & =\frac{12 \alpha_{X}^{2} B \tilde{m} m_{s}}{\left(\tilde{m}+2 m_{s}\right)+\left(2 \tilde{m}+m_{s}\right) \alpha_{X}^{2}+\sqrt{\left[\left(\tilde{m}+2 m_{s}\right)+\left(2 \tilde{m}+m_{s}\right) \alpha_{X}^{2}\right]^{2}-36 \alpha_{X}^{2} \tilde{m} m_{s}}} \\
m_{S_{2}}^{2} & =\left(\frac{6 A}{F_{\pi}^{2}}\right) \omega_{1}^{2}+\left(\frac{2 A}{F_{X}^{2}}\right)\left(1-\omega_{1}\right)^{2}+\frac{3 F_{X}^{2} \omega_{1}^{2}}{F_{\pi}^{2}\left(1-\omega_{1}\right)^{2}+3 F_{X}^{2} \omega_{1}^{2}} \frac{2}{3} B\left(2 \tilde{m}+m_{s}\right)
\end{aligned}
$$


where $\alpha_{X}^{2} \equiv \frac{F_{\pi}^{2}\left(1-\omega_{1}\right)^{2}}{F_{\pi}^{2}\left(1-\omega_{1}\right)^{2}+3 F_{X}^{2} \omega_{1}^{2}}$. Using the fact that $0 \leq \alpha_{X}^{2} \leq 1$ (and $\tilde{m}<m_{s}$ ), it is easy to show that:

$$
m_{S_{1}}^{2} \leq 2 B \tilde{m}=m_{\pi}^{2} \simeq(135 \mathrm{MeV})^{2}
$$

Even assuming, as already said, that we can identify the singlet $S_{2}$ with the observed singlet $\eta^{\prime}$, no other singlet pseudoscalar meson is observed whose mass satisfies the limit (B.9). Our assumption $c_{1}=0$ (together with $F_{X} \neq 0$ ) has thus led us to another " $U(1)$ problem". Even if we let $c_{1}$ be different from zero, but arbitrarily small, i.e., $c_{1} \rightarrow 0$ with all other quantities fixed, since, by virtue of Eqs. (2.11)-(2.12), the squared masses $m_{S_{1}, S_{2}}^{2}$ in the chiral limit are continuous functions of the parameter $c_{1}$, we find that $m_{S_{1}}^{2} \simeq Q_{L} / Z_{L} \simeq \frac{2 L c}{F_{\pi}^{2}\left(1-\omega_{1}\right)^{2}+L F_{X}^{2} \omega_{1}^{2}}=\mathcal{O}\left(c_{1}\right)$ will be arbitrarily small and, when including quark masses, it will have an upper limit arbitrarily close (from above) to that reported in Eq. (B.9).

Therefore, we are forced to discard this possibility (as it leads to wrong predictions for the pseudoscalar-meson mass spectrum) and, in the rest of this paper, we shall always consider the model in which $c_{1}$ is different from zero and not too small, so that $c=\mathcal{O}(N)$ is large. In this case, as we have seen in subsections 2.1 and 2.2, the squared masses of the singlet mesons $S_{1}$ and $S_{2}$ are given by Eq. (2.15) in the chiral limit and by Eqs. (2.30) and (2.31) in the realistic case with $L=3$ light quark flavours. Therefore, as already said, the state $S_{1}$ has a topological (non-chiral) squared mass of order $\mathcal{O}(1 / N)$ in the large- $N$ limit and it is nothing but the particle $\eta^{\prime}$. Instead, the state $S_{2}$ is identified with an exotic singlet particle $\eta_{X}$, having a large (non-chiral) mass term of order $\mathcal{O}(1)$ in the large $-N$ limit, generated by the (non-zero) coupling constant $c_{1}$. 


\section{References}

[1] S.B. Treiman, in "Lectures in Current Algebra and Its Applications", edited by S.B. Treiman, R. Jackiw, and D.J. Gross (Princeton University Press, Princeton, 1972); S.L. Adler and R.F. Dashen, "Current Algebras" (Benjamin, New York, 1968);

S. Coleman, "Aspects of symmetry" (Cambridge University Press, Cambridge, 1985).

[2] S. Weinberg, Phys. Rev. D 11, 3583 (1975).

[3] G. 'tHooft, Phys. Rev. Lett. 37, 8 (1976);

G. 'tHooft, Phys. Rev. D 14, 3432 (1976) [Erratum-ibid. D 18, 2199 (1978)].

[4] E. Witten, Nucl. Phys. B 156, 269 (1979).

[5] G. Veneziano, Nucl. Phys. B 159, 213 (1979).

[6] E. Meggiolaro, Z. Phys. C 62, 669 (1994).

[7] E. Meggiolaro, Z. Phys. C 62, 679 (1994).

[8] E. Meggiolaro, Z. Phys. C 64, 323 (1994).

[9] A. Di Giacomo and E. Meggiolaro, Nucl. Phys. B (Proc. Suppl.) 42, 478 (1995).

[10] E. Meggiolaro, "Remarks on the U(1) axial symmetry in QCD at zero and non-zero temperature", arXiv:hep-ph/0206236 (2002).

[11] M. Marchi and E. Meggiolaro, Nucl. Phys. B 665, 425 (2003).

[12] E. Meggiolaro, Phys. Rev. D 69, 074017 (2004).

[13] C. Bernard et al., Nucl. Phys. B (Proc. Suppl.) 53, 442 (1997);

C. Bernard et al., Phys. Rev. Lett. 78, 598 (1997).

[14] F. Karsch, Nucl. Phys. B (Proc. Suppl.) 83-84, 14 (2000);

P.M. Vranas, Nucl. Phys. B (Proc. Suppl.) 83-84, 414 (2000).

[15] M. Cheng et al., Eur. Phys. J. C 71, 1564 (2011).

[16] M. Kobayashi and T. Maskawa, Prog. Theor. Phys. 44, 1422 (1970). 
[17] T. Kunihiro, Prog. Theor. Phys. 122, 255 (2009).

[18] P. Di Vecchia and G. Veneziano, Nucl. Phys. B 171, 253 (1980).

[19] E. Witten, Annals of Physics 128, 363 (1980).

[20] C. Rosenzweig, J. Schechter, C.G. Trahern, Phys. Rev. D 21, 3388 (1980).

[21] K. Kawarabayashi and N. Ohta, Nucl. Phys. B 175, 477 (1980).

[22] P. Nath and R. Arnowitt, Phys. Rev. D 23, 473 (1981).

[23] P. Di Vecchia, F. Nicodemi, R. Pettorino, G. Veneziano, Nucl. Phys. B 181, 318 (1981).

[24] K. Nakamura et al. (Particle Data Group), J. Phys. G 37, 075021 (2010).

[25] M. Teper, Phys. Lett. B 202, 553 (1988);

M. Campostrini, A. Di Giacomo, Y. Günduc, M.P. Lombardo, H. Panagopoulos, R. Tripiccione, Phys. Lett. B 252, 436 (1990);

B. Allés, M. D’Elia, A. Di Giacomo, Nucl. Phys. B 494, 281 (1997) [Erratum-ibid. B 679, 397 (2004)].

[26] H. Leutwyler, Phys. Lett. B 374, 181 (1996).

[27] E. Witten, Nucl. Phys. B 160, 57 (1979).

[28] M. Gell-Mann, "The Eightfold Way: A Theory Of Strong Interaction Symmetry", California Institute of Technology Report CTSL-20 (1961);

M. Gell-Mann and Y. Ne'eman, "The Eightfold Way" (W.A. Benjamin, New York, 1964).

[29] S. Okubo, Prog. Theor. Phys. 27, 949 (1962);

S. Okubo, Prog. Theor. Phys. 28, 24 (1962).

[30] A.I. Davydychev and R. Delbourgo, J. Phys. A 37, 4871 (2004).

[31] J. Gasser and H. Leutwyler, Nucl. Phys. B 250, 539 (1985).

[32] J. Kambor, C. Wiesendanger, D. Wyler, Nucl. Phys. B 465, 215 (1996). 
[33] A.V. Anisovich and H. Leutwyler, Phys. Lett. B 375, 335 (1996).

[34] N. Beisert and B. Borasoy, Nucl. Phys. A 716, 186 (2003).

[35] B. Borasoy and R. Nißler, Eur. Phys. J. A 26, 383 (2005).

[36] J. Bijnens and K. Ghorbani, J. High Energy Phys. 0711, 030 (2007).

[37] C. Ditsche, B. Kubis, U.G. Meißner, Eur. Phys. J. C 60, 83 (2009).

[38] N.G. Deshpande and T.N. Truong, Phys. Rev. Lett. 41, 1579 (1978).

[39] H. Leutwyler, Nucl. Phys. Proc. Suppl. 64, 223 (1998).

[40] M.A. Shifman, A.I. Vainshtein, V.I. Zakharov, Nucl. Phys. B 147, 385, 448, 519 (1979).

[41] F. Giacosa, Phys. Rev. D 75, 054007 (2007).

[42] G. 'tHooft, G. Isidori, L. Maiani, A.D. Polosa, V. Riquer, Phys. Lett. B 662, 424 (2008).

[43] A.H. Fariborz, R. Jora, J. Schechter, Phys. Rev. D 77, 094004 (2008);

A.H. Fariborz, R. Jora, J. Schechter, Phys. Rev. D 79, 074014 (2009). 